\title{
VANISHING CRITICAL MAGNETIZATION IN THE QUANTUM ISING MODEL
}

\author{
JAKOB E. BJÖRNBERG
}

\begin{abstract}
Adapting the recent argument of Aizenman, DuminilCopin and Sidoravicius for the classical Ising model, it is shown here that the magnetization in the transverse-field Ising model vanishes at the critical point. The proof applies to the ground state in dimension $d \geq 2$ and to positive-temperature states in dimension $d \geq 3$, and relies on graphical representations as well as an infrared bound.
\end{abstract}

\section{INTRODUCTION}

This article concerns the transverse-field Ising model, introduced in [22] and a well-known generalization of the familiar (classical) Ising model for ferromagnetism. The model possesses a phase transition and a critical point, which may be identified using the (residual) magnetization. The magnetization equals zero below the critical point and is positive above it. An important result for the classical Ising model is that the magnetization also vanishes at the critical point: in two dimensions this goes back to the work of Onsager [23], in dimension $d \geq 4$ it was first proved by Aizenman and Fernández [3], and recently the final case $d=3$ was established by Aizenman, Duminil-Copin and Sidoravicius [2]. Building on the methods of [2], the present work shows that the magnetization in the transverse-field model also vanishes at the critical point. This implies that there is a unique equilibrium state at the critical point. We give precise statements shortly, but first introduce the relevant notation and definitions.

Let $n \geq 1$ and write

$$
\Lambda=\Lambda_{n}=[-n, n]^{d}=\{-n,-n+2, \ldots, n-1, n\}^{d}
$$

for a finite box in $\mathbb{Z}^{d}$. The transverse-field Ising model is defined via its Hamiltonian, which in the finite volume $\Lambda$ takes the form

$$
H_{\Lambda}=-\lambda \sum_{x y \in \Lambda} \sigma_{x}^{(3)} \sigma_{y}^{(3)}-\delta \sum_{x \in \Lambda} \sigma_{x}^{(1)}-\gamma \sum_{x \in \Lambda} \sigma_{x}^{(3)}
$$

Date: February 2, 2018.

Department of Mathematics, Uppsala University, Box 256, 75105 Uppsala, Sweden, Phone +46(0)18-471 3106, e-mail: jakob@math.uu.se. 
Here the first sum is over all (unordered) nearest neighbours in $\Lambda$,

$$
\sigma^{(1)}=\left(\begin{array}{cc}
1 & 0 \\
0 & -1
\end{array}\right), \quad \sigma^{(3)}=\left(\begin{array}{ll}
0 & 1 \\
1 & 0
\end{array}\right)
$$

are the spin- $\frac{1}{2}$ Pauli matrices, and $\sigma_{x}^{(i)}=\sigma^{(i)} \otimes \operatorname{Id}_{\Lambda \backslash\{x\}}$. The parameters $\lambda$ and $\delta, \gamma$ are nonnegative and represent spin-coupling and field-strengths, respectively. $H_{\Lambda}$ is an operator on the Hilbert space $\bigotimes_{x \in \Lambda} \mathbb{C}^{2}$, and one defines for each $\beta \in(0, \infty)$ the state $\langle\cdot\rangle_{\Lambda, \beta}$ by

$$
\langle Q\rangle_{\Lambda, \beta}=\frac{\operatorname{tr}\left(Q e^{-\beta H_{\Lambda}}\right)}{\operatorname{tr}\left(e^{-\beta H_{\Lambda}}\right)} .
$$

The parameter $\beta$ is referred to as inverse temperature. (Readers with a probabilistic background may prefer the 'path integral' definition of $\langle\cdot\rangle_{\Lambda, \beta}$ given in Section 2.1.)

Of particular interest are the one- and two-point functions

$$
\left\langle\sigma_{x}^{(3)}\right\rangle_{\Lambda, \beta} \quad \text { and } \quad\left\langle\sigma_{x}^{(3)} \sigma_{y}^{(3)}\right\rangle_{\Lambda, \beta},
$$

and more general correlation functions

$$
\left\langle\sigma_{A}^{(3)}\right\rangle_{\Lambda, \beta}, \quad \text { where } \sigma_{A}^{(3)}=\prod_{x \in A} \sigma_{x}^{(3)} .
$$

As written, these are analytical functions of the model parameters $\lambda, \delta, \gamma$. However, one is interested in their their limits as $n \rightarrow \infty$ or $\beta, n \rightarrow \infty$. (Existence of the limits is well-known, see eg [4] or [7].) These need not be analytical, or even continuous: for example if $\gamma=0$ then by symmetry $\left\langle\sigma_{0}^{(3)}\right\rangle_{\Lambda, \beta}=0$, whereas the residual magnetization

$$
\begin{aligned}
M_{\beta}^{+}(\lambda, \delta) & :=\lim _{\gamma \downarrow 0} \lim _{n \rightarrow \infty}\left\langle\sigma_{0}^{(3)}\right\rangle_{\Lambda, \beta} \\
\text { or } M_{\infty}^{+}(\lambda, \delta) & :=\lim _{\gamma \downarrow 0} \lim _{n \rightarrow \infty} \lim _{\beta \rightarrow \infty}\left\langle\sigma_{0}^{(3)}\right\rangle_{\Lambda, \beta}
\end{aligned}
$$

may be strictly positive. This leads to the definition of the critical point

$$
\lambda_{\mathrm{c}}=\lambda_{\mathrm{c}}(\delta, \beta):=\inf \left\{\lambda \geq 0: M_{\beta}^{+}(\lambda, \delta)>0\right\} .
$$

Note that $\lambda_{c}$ may also be defined in terms of the uniqueness of the infinite-volume states (for all boundary conditions), or the divergence of the susceptibility, see [9]. We have that $0<\lambda_{\mathrm{c}}<\infty$ if $d \geq 2$, or if $\beta=\infty$ and $d \geq 1$. The case $\beta=\infty$ is referred to as the ground state and the case $\beta<\infty$ as positive temperature.

The following is the main result of this work:

Theorem 1.1. Let $\delta>0$. If $\beta=\infty$ and $d \geq 2$, or $\beta<\infty$ and $d \geq 3$, then the residual magnetization satisfies

$$
M_{\beta}^{+}\left(\lambda_{\mathrm{c}}, \delta\right)=0 .
$$


If $\delta=0$ one recovers the classical Ising model; it is then standard to take $\lambda=1$ and vary the parameter $\beta$, giving the critical point $\beta_{\mathrm{c}}$ which is positive and finite if $d \geq 2$. As remarked above, in this case the result is well-known. For $\delta>0$ the case when $\beta=\infty$ and $d=1$ is also known and was established in [24] (and reproved in [9] using graphical methods). The other cases are new. Note that the only nontrivial case left open by Theorem 1.1 is when $\beta<\infty$ and $d=2$, which remains open for $\delta>0$.

Like the previous works [2, 3] on the classical model, our proof of Theorem 1.1 uses graphical representations. For the classical Ising model, and related models such as the Potts model, the use of graphical representations is a standard tool and has been a huge success since the seminal work of Fortuin and Kasteleyn [17]. In more recent times graphical representations have also been very successful in the study of quantum models, not only the Ising model [4, 8, 9, 12, 15] but also Heisenberg models [5, 25, 26] (see also [19]). The transverse-field Ising model possesses at least three graphical representations, which may be called firstly the space-time spin representation, secondly the randomparity representation, and thirdly the FK-representation. The first of these goes back to [15], the last to to [4, 12], whereas the second was developed in [9] (see also [13] for the related random-current representation). These graphical representations are obtained by applying the Lie-Trotter expansion to the correlations functions (2) using the eigenbasis for either $\sigma^{(3)}$ or $\sigma^{(1)}$, see [20].

Of primary importance for the present work is the random-parity representation, which is described in Section 2.2. It is a continuous version of the random-current representation for the classical Ising model, developed in [1. The key insight of the works [1, 2] was that the phase transition in the (classical) Ising model relates to a percolation transition for the random currents, and the present work exploits a similar picture for the quantum model.

In addition to graphical representations, the other key component of the proof of Theorem [1.1 is an infrared bound proved in [8], and stated below in (26). This is a bound on the Fourier-transform of the Schwinger function:

$$
c((x, s),(y, t))=\frac{1}{\operatorname{tr}\left(e^{-\beta H_{\Lambda}}\right)} \operatorname{tr}\left(e^{-(\beta-t+s) H_{\Lambda}} \sigma_{y}^{(3)} e^{-(t-s) H_{\Lambda}} \sigma_{x}^{(3)}\right) .
$$

Infrared bounds go back to [18] and one of their great successes is the proof by Dyson, Lieb and Simon [16] of the existence of a phase transition in the antiferromagnetic Heisenberg model. See also [26] for a recent infrared bound for the Heisenberg model in the same spirit as the bound employed here.

The argument for proving Theorem 1.1 follows the general outline of the argument for the classical model given in [2]. The first step is 
to develop an infinite-volume version of the random-parity representation and study percolation under this measure, see Section 3 . The infrared bound is used to show that when $\lambda=\lambda_{\text {c }}$ then (for $\beta<\infty$ and $d \geq 3$ or $\beta=\infty$ and $d \geq 2$ ) there is no unbounded percolation cluster, see Proposition 4.2. Combined with 'local modifcations' of the random-parity representation (Proposition 2.2) and the switching lemma (Lemma 2.1), this allows us to deduce the result, as described in Section 4. Compared with the classical model [2], the main difficulty in the present work arises from the 'continuous' nature of the graphical representations in the quantum setting. For example, the configuration space of the random-parity representation is non-compact so an argument is needed to obtain tightness of the sequence of finite-volume random-parity measures (see Proposition 3.1). Related difficulties arise in proving insertion tolerance (see Proposition 2.2) and ergodicity (see Lemma 5.1).

In the rest of this article we fix $\delta>0$. We also set $\gamma=0$ and use the equality of the residual and spontaneous magnetization (91). We use the abbreviation TFIM for transverse-field Ising model, and we use the following probabilistic notation: $\mathbb{I}_{A}$ or $\mathbb{I}\{A\}$ for the indicator function taking value 1 if the event $A$ occurs, 0 otherwise, and $\mathbb{P}(X)$ for the expectation of the random variable $X$ under the probability measure $\mathbb{P}$.

\section{Graphical Representations}

In this section we present two graphical representations of the TFIM, namely the space-time spin representation and the random-parity representation. The latter represents the correlation functions (2) and Schwinger functions (4) in terms of expectations over random 'paths' and is of central importance to this work. A major technical tool in this representation is the switching lemma, which we describe in Section 2.2.1. In Section 2.2.2 we then prove some results on 'local modifications' in this representation, which are forms of insertion- and deletion tolerance.

The space-time spin representation has a less prominent role in the main argument than the random-parity representation, and is used mainly to establish an ergodicity property of the infinite-volume randomparity representation (see Lemma 5.1 and Proposition 3.2 ). However, it provides a natural setting to introduce a class of boundary conditions that are of central importance to this work, and may also provide a more intuitive description of the TFIM to readers with a probabilistic background than the definition given in Section 1.

Before proceeding we introduce some notation. Recall that $\Lambda_{N}$ denotes the box $[-N, N]^{d} \subseteq \mathbb{Z}^{d}$ and let $\partial \Lambda_{N}=\Lambda_{N} \backslash \Lambda_{N-1}$ denote the boundary of $\Lambda_{N}$. For $r>0$ write $I_{r}$ for the interval $[-r / 2, r / 2] \subseteq \mathbb{R}$ and define $K(N, r)=\Lambda_{N} \times I_{r}$. Also write $I_{\infty}=\mathbb{R}, \mathbb{K}_{\beta}=\mathbb{Z}^{d} \times I_{\beta}$ and 
$\mathbb{K}=\mathbb{K}_{\infty}=\mathbb{Z}^{d} \times I_{\infty}$. We frequently think of $\mathbb{K}$ as a subset of $\mathbb{R}^{d+1}$ and $K(N, r)$ as a subset of $\mathbb{K}$ in the natural way. For elements $x \in \mathbb{Z}^{d}$ or $(x, t) \in \mathbb{K}$ we write $\|x\|$ and $\|(x, t)\|=\|x\|+|t|$ for their $\ell^{1}$-norm. We will also use the notation $\mathcal{E}=\left\{x y: x, y \in \mathbb{Z}^{d},\|x-y\|=1\right\}$ for the set of unordered pairs of nearest neighbours in $\mathbb{Z}^{d}, \mathcal{E}_{N}=\{x y: x, y \in$ $\left.\Lambda_{N},\|x-y\|=1\right\}$ for nearest neighbours in $\Lambda_{N}$, and $F(N, r)=\mathcal{E}_{N} \times I_{r}$ as well as $\mathbb{F}=\mathcal{E} \times I_{\infty}$.

2.1. Space-time spin representation. Let $\Sigma_{\beta}$ be the set of functions $\sigma(\cdot, \cdot): \mathbb{K}_{\beta} \rightarrow\{-1,+1\}$ such that for all $x \in \mathbb{Z}^{d}$, the restriction $\sigma(x, \cdot): I_{\beta} \rightarrow\{-1,+1\}$ is right-continuous and changes value finitely often in each bounded interval. Also let $\Sigma=\Sigma_{\infty}$ and let $\Sigma(N, r)$ be the set of restrictions of elements of $\Sigma$ to $K(N, r)$. The space-time spin representation is based on probability measures on $\Sigma(N, r)$. To define these, let $E$ be a probability measure governing

(a) a collection $D=\left(D_{x}: x \in \mathbb{Z}^{d}\right)$ of independent Poisson processes on $\mathbb{R}$ of rate $\delta$, and

(b) a collection $\xi=\left(\xi_{x}: x \in \mathbb{Z}^{d}\right)$ of independent random variables taking the values 0 or 1 with equal probability.

We let

$$
\sigma(x, t)= \begin{cases}(-1)^{\xi_{x}+\left|D_{x} \cap(0, t]\right|} & \text { if } t \geq 0 \\ (-1)^{\xi_{x}+\left|D_{x} \cap(t, 0]\right|} & \text { if } t<0 .\end{cases}
$$

Thus $\sigma(x, 0)=(-1)^{\xi_{x}}$ and $\sigma(x, t)$ switches value at the points $t \in D_{x}$. See Figure 1 for an example. We sometimes write $\sigma_{x, t}$ for $\sigma(x, t)$. In this way $E$ defines an 'a-priori' measure on $\Sigma$. We write $E_{N, r}$ for the induced measure on $\Sigma(N, r)$.

The general notation for space-time Ising probability measures on $\Sigma(N, r)$ will be of the form

$$
\mu_{N, r}^{\mathfrak{s}, \mathfrak{t}}(\cdot), \quad \text { where } \mathfrak{s}, \mathfrak{t} \in\{\mathfrak{f}, \mathfrak{w}, \mathfrak{p}\} .
$$

The superscripts $\mathfrak{s}, \mathfrak{t}$ denote boundary conditions, 'spatial' and 'temporal', respectively, and their values $\mathfrak{f}, \mathfrak{w}, \mathfrak{p}$ stand for 'free', 'wired' and 'periodic'. The easiest to describe is $\mu_{N, r}^{\mathfrak{f}, \mathfrak{f}}$, which is given by its density

$$
\frac{d \mu_{N, r}^{\mathfrak{f}, \mathfrak{f}}}{d E_{N, r}}(\sigma)=\frac{1}{Z_{N, r}^{\mathfrak{f}, \mathfrak{f}}} \exp \left(\lambda \sum_{x y \in \mathcal{E}_{N}} \int_{I_{r}} \sigma(x, t) \sigma(y, t) d t\right) .
$$

Here

$$
Z_{N, r}^{\mathfrak{f}, \mathfrak{f}}=E_{N, r}\left[\exp \left(\lambda \sum_{x y \in \mathcal{E}_{N}} \int_{I_{r}} \sigma(x, t) \sigma(y, t) d t\right)\right]
$$

is the appropriate normalization. To obtain the boundary conditions $\mathfrak{t}=\mathfrak{w}$ and $\mathfrak{t}=\mathfrak{p}$, respectively, we include in the density (15) (and in the partition function (6) $)$ the following restrictions:

- for $\mathfrak{t}=\mathfrak{w}$, that $\sigma(x,-r / 2)=\sigma(x, r / 2)=+1$ for all $x \in \Lambda_{N}$,

- for $\mathfrak{t}=\mathfrak{p}$, that $\sigma(x,-r / 2)=\sigma(x, r / 2)$ for all $x \in \Lambda_{N}$. 

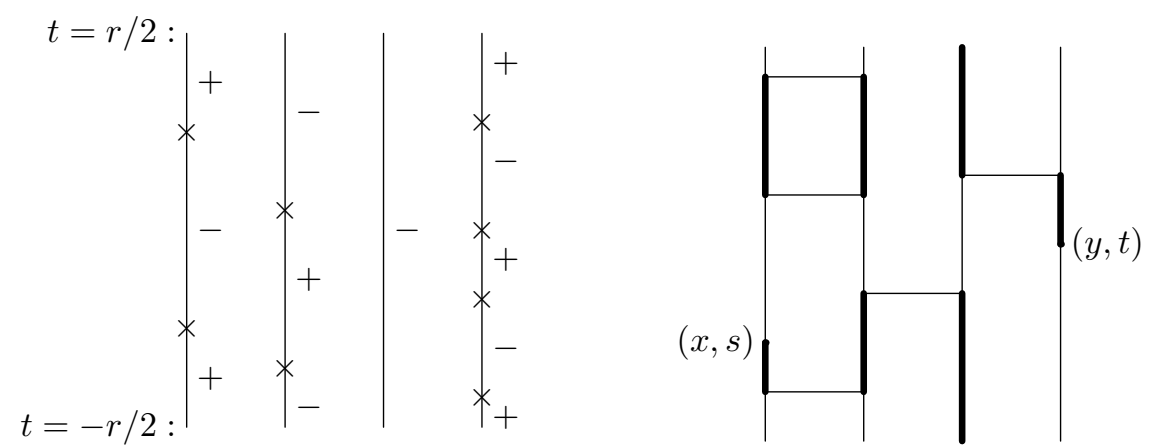

Figure 1. Left: A realization of the spin-representation $\sigma$. The elements of $D$ are marked as $\times$, and the value of $\sigma$ is indicated next to each line segment. (This realization is compatible with $\mathfrak{t}=\mathfrak{f}$ or $\mathfrak{p}$ but not with $\mathfrak{t}=\mathfrak{w}$.) Right: A realization of the random-parity representation $\psi_{A}^{\mathfrak{p}}$, where $A=\{(x, s),(y, t)\}$. 'Odd' intervals are drawn bold, bridges as horizontal lines. (This realization is not compatible with $\mathfrak{t}=\mathfrak{f}$ or $\mathfrak{w}$.)

(Thus, intuitively, for $\mathfrak{t}=\mathfrak{w}$ the spins at the endpoints of $I_{r}$ are 'frozen' to be +1 , whereas for $\mathfrak{t}=\mathfrak{p}$ we think of $I_{r}$ as a circle of length $r$.) To obtain the boundary condition $\mathfrak{s}=\mathfrak{w}$ we replace $\mathcal{E}_{N}$ by $\mathcal{E}_{N+1}$ in the sums in (51)-(6) and set $\sigma(x, t)=+1$ for any $x \in \partial \Lambda_{N+1}$. Finally, to obtain the boundary condition $\mathfrak{s}=\mathfrak{p}$ we replace $\mathcal{E}_{N}$ by the set $\mathcal{E}_{N}^{\mathfrak{p}}$ obtained by adding to $\mathcal{E}_{N}$ all pairs $x y$ such that $x$ and $y$ differ in exactly one coordinate, this coordinate being $-N$ in one case and $N$ in the other. (Intuitively this makes $\Lambda_{N}$ 'wrap around' in each coordinate direction.)

The connection to the TFIM is that the correlation functions (2) satisfy

$$
\left\langle\sigma_{A}^{(3)}\right\rangle_{\Lambda, \beta}=\mu_{n, \beta}^{\mathfrak{f}, \mathfrak{p}}\left(\prod_{x \in A} \sigma(x, 0)\right),
$$

and the Schwinger function (41)

$$
c((x, s),(y, t))=\mu_{n, \beta}^{\mathfrak{f}, \mathfrak{p}}(\sigma(x, s) \sigma(y, t)) .
$$

(Recall that we write $\mu(f)$ for the $\mu$-expectation of a function $f(\sigma)$.) In light of this correspondence it is natural to use the notation

$$
\left\langle\sigma_{A}\right\rangle_{N, r}^{\mathfrak{s , t}}=\mu_{N, r}^{\mathfrak{s}, \mathrm{t}}\left(\prod_{(x, t) \in A} \sigma(x, t)\right)
$$

for general finite subsets $A \subseteq K(N, r)$. Henceforth we refer to the quantities in (7) as correlation functions.

The $\mathfrak{p}$-boundary condition in 'time' arises automatically from the cyclicity of the trace in (1). The other boundary conditions $\mathfrak{t}=\mathfrak{f}, \mathfrak{w}$ are convenient when working with infinite-volume limits. The correlation 
functions (7) have a natural monotonicity in the boundary conditions. In particular,

$$
\left\langle\sigma_{A}\right\rangle_{N, r}^{\mathfrak{f}, \mathfrak{f}} \leq\left\langle\sigma_{A}\right\rangle_{N, r}^{\mathfrak{f}, \mathfrak{p}} \leq\left\langle\sigma_{A}\right\rangle_{N, r}^{\mathfrak{p}, \mathfrak{p}} \leq\left\langle\sigma_{A}\right\rangle_{N, r}^{\mathfrak{w}, \mathfrak{p}} \leq\left\langle\sigma_{A}\right\rangle_{N, r}^{\mathfrak{w}, \mathfrak{w}}, \text { etc. }
$$

(A detailed proof for the present model may easily be devised using Theorem 2.2.12 and Lemma 2.2.21 of [7].)

When $\beta<\infty$ we fix $\mathfrak{t}=\mathfrak{p}$ and work with the measures $\mu_{N, \beta}^{\mathfrak{f}, \mathfrak{p}}$ and $\mu_{N, \beta}^{\mathfrak{w}, \mathfrak{p}}$. When $\beta=\infty$ we will primarily be working with $\mu_{N, r}^{\mathfrak{f}, \mathfrak{f}}$ and $\mu_{N, r}^{\mathfrak{w}, \mathfrak{w}}$ where $r=2 N$. The (weak) limits of these measures exist as $N \rightarrow \infty$ and are related, respectively, to the positive-temperature and ground-state limits appearing in (3). In particular,

(9) $M_{\infty}^{+}=\lim _{N \rightarrow \infty}\langle\sigma(0,0)\rangle_{N, r=2 N}^{\mathfrak{w}, \mathfrak{w}}, \quad M_{\beta}^{+}=\lim _{N \rightarrow \infty}\langle\sigma(0,0)\rangle_{N, \beta}^{\mathfrak{w}, \mathfrak{p}}$ for $\beta<\infty$,

i.e. the residual and spontaneous magnetization coincide, cf. 21 and Section 2.5.2 of [7]. We write $\mu_{\lambda, \beta}^{(\mathfrak{s}, \mathfrak{t})}(\cdot)$ or $\langle\cdot\rangle_{\lambda, \beta}^{(\mathfrak{s}, \mathfrak{t})}$ for any weak limit obtained as above with boundary conditions $\mathfrak{s}, \mathfrak{t}$ (and $\beta<\infty$ or $\beta=$ $\infty)$. When $M^{+}=0$ then the limit measure is unique:

$$
\mu_{\lambda, \beta}^{(\mathfrak{f}, \mathfrak{p})}=\mu_{\lambda, \beta}^{(\mathfrak{w}, \mathfrak{p})} \text { for } \beta<\infty, \quad \mu_{\lambda, \infty}^{(\mathfrak{f}, \mathfrak{f})}=\mu_{\lambda, \infty}^{(\mathfrak{w}, \mathfrak{w})} .
$$

(A detailed proof for the present model appears in [7, Theorem 2.5.9]; it follows closely the argument for the classical model [21].) Thus our main result Theorem 1.1 implies that the limit measure is unique at the critical point. For more information about the statements in this subsection, and the spin-representation in general, see [7.

2.2. Random-parity representation. Like the space-time spin representation of the previous subsection, the random-parity representation expresses the correlation functions (7) using Poisson processes in $K(N, r)$. This time we write $E_{N, r}$ for a probability measure governing a collection $B=\left(B_{x y}: x y \in \mathcal{E}_{N}\right)$ of independent Poisson processes on $I_{r}$ with intensity $\lambda$, as well as an independent collection $\tau=\left(\tau_{x}: x \in \Lambda_{N}\right) \in\{0,1\}^{\Lambda_{N}}$ of independent random variables taking values 0 or 1 with equal probability $1 / 2$. We sometimes refer to the points of $B$ as bridges. When considering the correlation function $\left\langle\sigma_{A}\right\rangle_{N, r}^{\mathfrak{s , t}}$ we will use the term switching point for any point $(x, t) \in K(N, r)$ such that either (i) $(x, t) \in A$, or (ii) there exists $y \in \Lambda_{N}$ such that $t \in B_{x y}$ (ie, $(x, t)$ is the 'endpoint' of some bridge). The collection of switching points is denoted by $S=\left(S_{x}: x \in \Lambda_{N}\right)$, where $S_{x}$ is the set of $t \in I_{r}$ such that $(x, t)$ is a switching point. The points of $A$ are referred to as sources. We say that $B$ is consistent with $A$ if $\left|S_{x}\right|$ is even for each $x$, and in this case we will also refer to $S$ itself as consistent.

For each consistent $S$ we will define a labelling of $K(N, r)$ using the labels 'even' and 'odd', and for definiteness we use the convention that the 'odd' subset is closed. See Figure 1 again for an illustration of the 
description that follows. The definition will depend on the boundary conditions $\mathfrak{s}$ and $\mathfrak{t}$. We will not be using the random-parity representation for $\mathfrak{s}=\mathfrak{p}$ so we omit describing it. For $\mathfrak{s}=\mathfrak{f}$ we will denote the labelling $\psi_{A}^{\mathfrak{t}}$ and for $\mathfrak{s}=\mathfrak{w}$ by $\hat{\psi}_{A}^{\mathrm{t}}$. In what follows we assume for simplicity that $A$ does not contain any point of the form $(x, \pm r / 2)$. We begin with the case $\mathfrak{s}=\mathfrak{f}$ :

- If $\mathfrak{t}=\mathfrak{f}$ we label each point $(x, \pm r / 2)$ 'even', and define the rest of $\psi_{A}^{\mathfrak{f}}$ by requiring that labels switch between 'even' and 'odd' at the points of $S$ and are constant in between. This is possible due to the assumption that $S$ is consistent.

- If $\mathfrak{t}=\mathfrak{w}$ we instead label all points of the form $(x, \pm r / 2)$ 'odd' and apply the same rule for switching at points of $S$.

- If $\mathfrak{t}=\mathfrak{p}$ we require the vector $\tau$. We define $\psi_{A}^{\mathfrak{p}}$ by labelling each $(x, 0)$ 'even' if $\tau_{x}=0$ or 'odd' if $\tau_{x}=1$, and letting the label switch at the points of $S$ as before. Due to the consistency of $S$ we can think of $\psi_{A}^{\mathfrak{p}}$ as a labelling of the circle. (Clearly the choice $(x, 0)$ is arbitrary, and one may equally well let $\tau$ determine all the labels $(x, t)$ for any fixed $t$.)

We now describe how to define the labelling in the case when $\mathfrak{s}=\mathfrak{w}$. We now let $\hat{E}_{N, r}$ denote a probability measure which, in addition to processes $B$ and $\tau$ as above, also governs a process $G=\left(G_{x}: x \in \partial \Lambda_{N}\right)$ of independent Poisson processes on $I_{r}$. The intensity of $G_{x}$ depends on $x$, and equals $\lambda$ times the number of $y \in \mathbb{Z}^{d} \backslash \Lambda_{N}$ such that $x y \in \mathcal{E}$. For simplicity we set $G_{x}=\varnothing$ for $x \in \Lambda_{N} \backslash \partial \Lambda_{N}$. We augment the switching points $S$ to contain also each $(x, t)$ such that $t \in G_{x}$. As before we say that $S$ is consistent if each $\left|S_{x}\right|$ is even, and in that case we also say that the pair $(B, G)$ is consistent with $A$. Given a consistent $S$ we obtain the labelling, which we now denote $\hat{\psi}_{A}^{\mathrm{t}}$, precisely as before.

Given a labelling $\psi_{A}^{\mathrm{t}}$ (respectively, $\hat{\psi}_{A}^{\mathrm{t}}$ ), let $\epsilon$ denote the total length of all intervals in $K(N, r)$ labelled 'even', and let the weight $\partial \psi_{A}^{\mathrm{t}}$ (respectively, $\partial \hat{\psi}_{A}^{\mathrm{t}}$ ) be defined as $e^{2 \delta \epsilon}$. In the case when $S$ is not consistent we define the weight to be zero. The random-parity representation allows us to write

$$
\left\langle\sigma_{A}\right\rangle_{N, r}^{\mathfrak{f}, \mathfrak{t}}=\frac{E_{N, r}\left(\partial \psi_{A}^{\mathfrak{t}}\right)}{E_{N, r}\left(\partial \psi_{\varnothing}^{\mathrm{t}}\right)} \quad \text { and } \quad\left\langle\sigma_{A}\right\rangle_{N, r}^{\mathfrak{w}, \mathrm{t}}=\frac{\hat{E}_{N, r}\left(\partial \hat{\psi}_{A}^{\mathrm{t}}\right)}{\hat{E}_{N, r}\left(\partial \hat{\psi}_{\varnothing}^{\mathrm{t}}\right)} .
$$

The first of these $(\mathfrak{s}=\mathfrak{f})$ was proved in [9, Theorem 3.1], and the second $(\mathfrak{s}=\mathfrak{w})$ follows using similar arguments.

In fact, the representation (10) holds for correlations in more general subsets $K^{\prime}$ of $\mathbb{K}$ than $K(N, r)$, in particular for 'regions with holes'. We briefly outline this now (it will be used in Proposition 3.1). Write $K=$ $K(N, r)$, let $J$ be a finite collection of disjoint closed intervals in $K$, and let $K^{\prime}=K \backslash J$. Write $\partial J$ for the set of all endpoints of intervals in $J$ (if $J$ contains some interval consisting of a single point $(x, t)$ we distinguish 
between $(x, t+)$ and $(x, t-))$. For each $x y \in \mathcal{E}_{N}$ let $B_{x y}$ denote a Poisson process on $I_{r}$ with variable intensity: $\lambda$ if $(x, t),(y, t) \in K^{\prime}$, otherwise 0 . We start by labelling each point $(x, t) \in \partial J$ 'even'. If $\mathfrak{t}=\mathfrak{f}$ (respectively, $\mathfrak{t}=\mathfrak{w})$ we label each point $(x, \pm r / 2) \in K^{\prime}$ 'even' (respectively, 'odd'). If $\mathfrak{t}=\mathfrak{p}$ then for each $x$ such that $\{x\} \times I_{r} \subseteq K^{\prime}$ we let $\tau_{x}$ determine the label $(x, 0)$ as before. The full labelling is finally obtained by letting the labels switch at the points of $S$ as before. (A labelling $\hat{\psi}_{A}^{\mathrm{t}}$ of $K^{\prime}$ is obtained similarly, modifying also $G$ to have zero intensity in $J$.) Writing $E_{K^{\prime}}$ for the corresponding probability measure, we note that

$$
E_{K^{\prime}}\left(\partial \psi_{\varnothing}^{\mathfrak{t}}\right)=e^{-2 \delta|J|} E_{N, r}\left(\partial \psi_{\varnothing}^{\mathfrak{t}} \mathbb{I}\left\{\psi_{\varnothing}^{\mathfrak{t}} \text { 'even' in } J\right\}\right),
$$

where $|J|$ denotes the total length of the intervals comprising $J$, see [9].

2.2.1. Switching lemma. Consider the case in (10) when $A$ consists of the two points $(0,0)$ and $(x, t)$. The consistency constraint on $S$, and the way the labels are defined, forces the existence of an 'odd path' between the two sources $(0,0)$ and $(x, t)$. A similar, but more complicated, picture arises for general sets $A$. The main virtue of the random-parity representation is that this picture can be developed to the case of pairs of labellings in a way which also allows the representation of differences between correlation functions. The tool for this is called the switching lemma.

Let $\mathbb{E}_{N, r}$ denote a probability measure governing the following independent random variables:

(a) two copies of the process $B$, denoted $B$ and $\hat{B}$;

(b) two copies of $\tau$, denoted $\tau$ and $\hat{\tau}$;

(c) one copy of the process $G$; and

(d) one copy of a process $\Delta=\left(\Delta_{x}: x \in \Lambda_{N}\right)$, where the $\Delta_{x}$ are independent Poisson processes on $I_{r}$ with intensity $4 \delta$.

Thus we may write $\mathbb{E}_{N, r}=E_{N, r} \times \hat{E}_{N, r} \times P_{\Delta}$, where $P_{\Delta}$ denotes the distribution of $\Delta$. We call $\Delta$ the process of 'cuts'. If we fix two finite sets $A_{1}$ and $A_{2}$ of sources and two boundary conditions $\mathfrak{t}_{1}$ and $\mathfrak{t}_{2}$ then we obtain under $\mathbb{E}_{N, r}$ a triple $\left(\psi_{A_{1}}^{\mathfrak{t}_{1}}, \hat{\psi}_{A_{2}}^{\mathfrak{t}_{2}}, \Delta\right)$ whose components are independent. In what follows we will only be using the following combinations of boundary conditions $\mathfrak{t}_{1}, \mathfrak{t}_{2}$ : in the case $\beta<\infty$ we take $\mathfrak{t}_{1}=\mathfrak{t}_{2}=\mathfrak{p}$, and in the case $\beta=\infty$ we take $\mathfrak{t}_{1}=\mathfrak{f}$ and $\mathfrak{t}_{2}=\mathfrak{w}$, cf. the discussion below (8). In the latter case we use the notation $\hat{G}_{x}=G_{x} \cup\{-r / 2, r / 2\}$.

We next define a notion of open paths. The precise definition of a path depends on the boundary conditions $\mathfrak{t}_{1}, \mathfrak{t}_{2}$, and we start with the case $\mathfrak{t}_{1}=\mathfrak{f}, \mathfrak{t}_{2}=\mathfrak{w}$. If $\kappa, \kappa^{\prime} \in K(N, r)$ then an (open) path from $\kappa$ to $\kappa^{\prime}$ is a sequence of points $\kappa_{0}, \kappa_{1}, \ldots, \kappa_{2 m+1} \in K(N, r)$ satisfying the following:

(1) $\kappa_{0}=\kappa$ and $\kappa_{2 m+1}=\kappa^{\prime}$; 
(2) for each $j \in\{0, \ldots, m\}$, if $\kappa_{2 j}=(x, s)$ then $\kappa_{2 j+1}=(x, t)$ for some $t$ such that the interval $[s \wedge t, s \vee t]$ contains no point $s^{\prime} \in \Delta_{x}$ which is labelled 'even' in both labellings $\psi_{A_{1}}^{\mathfrak{f}}$ and $\hat{\psi}_{A_{2}}^{\mathfrak{w}}$;

(3) for each $j \in\{0, \ldots, m-1\}$, if $\kappa_{2 j+1}=(x, s)$ and $\kappa_{2 j+2}=(y, t)$ then either (i) $s=t \in B_{x y} \cup \hat{B}_{x y}$, or (ii) $s \in \hat{G}_{x}$ and $t \in \hat{G}_{y}$.

Intuitively this means that paths can traverse bridges, 'jump between' arbitrary points of $\hat{G}$, and traverse subintervals of $K(N, r)$, but are blocked by points of $\Delta$ which fall where both labellings are 'even'. One way to think of the 'jumping' between points of $\hat{G}$ is that the points in $\hat{G}$ are connections to and from a 'ghost-site' $\Gamma$.

To obtain the case $\mathfrak{t}_{1}=\mathfrak{t}_{2}=\mathfrak{p}$ we modify (ii) in item (3) above by replacing $\hat{G}$ with $G$ in both places, and we additionally allow in (2) that one may traverse either the inverval $[s, t]$ or the interval $[t, s]$, where these are to be regarded as intervals in the circle (we keep the restriction on $\Delta$ ). Intuitively these changes mean that the 'endpoints' $(x, \pm r / 2)$ are no longer connected to $\Gamma$ but are identified with each other.

The event that there is an open path between $\kappa$ and $\kappa^{\prime}$ is written $\left\{\kappa \leftrightarrow \kappa^{\prime}\right\}$. The possibility (ii) in (3) of 'jumping' via $\Gamma$ is special, and in some cases we want to consider paths which do not do this. We write $\left\{\kappa \leftrightarrow \kappa^{\prime}\right.$ off $\left.\Gamma\right\}$ for the event that there is some path that does not feature a pair $\kappa_{2 j+1}=(x, s), \kappa_{2 j+2}=(y, t)$ with $x \neq y$ and $s, t \in \hat{G}$. Note that when $\mathfrak{t}_{1}=\mathfrak{f}, \mathfrak{t}_{2}=\mathfrak{w}$ this also excludes jumping via the endpoints $(x, \pm r / 2)$. We also write $\{\kappa \leftrightarrow \Gamma\}$ for the event that some open path connects $\kappa$ to a point in $\hat{G}$ (respectively, $G$ ).

Write 0 for the origin $(0,0)$ and $\kappa$ for an arbitrary point in $K(N, r)$. We will be using the following form of the switching lemma:

LEMMA 2.1. $\mathbb{E}_{N, r}\left(\partial \psi_{0 \kappa}^{\mathrm{t}_{1}} \partial \hat{\psi}_{\varnothing}^{\mathrm{t}_{2}}\right)=\mathbb{E}_{N, r}\left(\partial \psi_{\varnothing}^{\mathrm{t}_{1}} \partial \hat{\psi}_{0 \kappa}^{\mathrm{t}_{2}} \mathbb{I}\{0 \leftrightarrow \kappa\right.$ off $\left.\Gamma\}\right)$.

Here we have abbreviated $\{0, \kappa\}$ with $0 \kappa$. The proof of Lemma 2.1 is a small modification of [9, Theorem 4.2], which we briefly outline now.

Proof sketch. Note that in the left-hand-side, the event $\{0 \leftrightarrow x$ off $\Gamma\}$ holds since there is an odd path in $\psi_{0 \kappa}^{\mathrm{t}_{1}}$. Condition on the sets $\bar{B}=B \cup \hat{B}$ and $G$, and note that the conditional distribution of the pair $(B, \hat{B})$ is given by assigning each element of $\bar{B}$ to $B$ or $\hat{B}$ with equal probability, independently. Given $\bar{B}$ and $G$ there is a finite collection of 'possible' paths $\pi_{1}, \ldots, \pi_{n}$ between 0 and $\kappa$ off $\Gamma$ (the numbering is arbitrary but fixed, and as noted above the collection is nonempty). When we assign the elements of $\bar{B}$ to $B$ and $\hat{B}$ and also sample $\Delta$ and (in the case $\left.\mathfrak{t}_{1}=\mathfrak{t}_{2}=\mathfrak{p}\right) \tau, \hat{\tau}$, this will both determine the labellings $\psi_{0 \kappa}^{\mathfrak{t}_{1}}$ and $\hat{\psi}_{\varnothing}^{\mathrm{t}_{2}}$, as well as reveal which of the possible paths are indeed open. Let $\pi_{j}$ be the first of these. Write $\psi_{0 \kappa}^{\mathrm{t}_{1}} \triangle \pi_{j}$ and $\hat{\psi}_{\varnothing}^{\mathrm{t}_{2}} \triangle \pi_{j}$ for the labellings obtained by switching 'even' and 'odd' along $\pi_{j}$. It is easy to see that 
these new labellings are consistent with sources $\varnothing$ and $0 \kappa$, respectively, and that they can be obtained by switching certain elements between $B$ and $\hat{B}$ as well as certain values of $\tau$ and $\hat{\tau}$. By symmetry, the latter transformations are measure-preserving. Moreover, it may be checked as in [9, p. 251] that the change in weight of the labellings exactly corresponds to the change in probability that $\pi_{j}$ is indeed the first open path. In particular, in the new configuration there is still a path between 0 and $\kappa$ off $\Gamma$.

The following simple application of Lemma 2.1 will be used in the proof of Theorem 1.1. We have that

$$
\begin{aligned}
\mathbb{E}_{N, r}\left(\partial \psi_{\varnothing}^{\mathrm{t}_{1}} \partial \hat{\psi}_{0 \kappa}^{\mathrm{t}_{2}}\right)-\mathbb{E}_{N, r}\left(\partial \psi_{0 \kappa}^{\mathrm{t}_{1}} \partial \hat{\psi}_{\varnothing}^{\mathrm{t}_{2}}\right) & =\mathbb{E}_{N, r}\left(\partial \psi_{\varnothing}^{\mathrm{t}_{1}} \partial \hat{\psi}_{0 \kappa}^{\mathrm{t}_{2}}[1-\mathbb{I}\{0 \leftrightarrow \kappa \text { off } \Gamma\}]\right) \\
& \leq \mathbb{E}_{N, r}\left(\partial \psi_{\varnothing}^{\mathrm{t}_{1}} \partial \hat{\psi}_{0 \kappa}^{\mathrm{t}_{2}} \mathbb{I}\{0 \leftrightarrow \Gamma\}\right) .
\end{aligned}
$$

In the last step we used that $\hat{\psi}_{0 \kappa}^{\mathrm{t}_{2}}$ contains an odd path $\pi$ between 0 and $\kappa$, and if there is no path between 0 and $\kappa$ off $\Gamma$ then $\pi$ must pass $\Gamma$. Combined with (10) this has the following consequence. Writing 0 for $(0,0)$ and $\kappa$ for $(x, t)$ we have from (12)

$$
0 \leq\left\langle\sigma_{0,0} \sigma_{x, t}\right\rangle_{N, r}^{\mathfrak{w}, \mathfrak{t}_{2}}-\left\langle\sigma_{0,0} \sigma_{x, t}\right\rangle_{N, r}^{\mathfrak{f}, \mathfrak{t}_{1}} \leq \frac{\mathbb{E}_{N, r}\left(\partial \psi_{\varnothing}^{\mathfrak{t}_{1}} \partial \hat{\psi}_{0 \kappa}^{\mathfrak{t}_{2}} \mathbb{I}\{0 \leftrightarrow \Gamma\}\right)}{\mathbb{E}_{N, r}\left(\partial \psi_{\varnothing}^{\mathfrak{t}_{1}} \partial \hat{\psi}_{\varnothing}^{\mathfrak{t}_{2}}\right)}
$$

2.2.2. Local modifications. Of central importance to the proof of Theorem 1.1 is a probability measure $\overline{\mathbb{P}}_{N, r}$ defined as follows. Recall the processes $B, \hat{B}, G, \Delta, \tau, \hat{\tau}$ as well as the labellings $\psi, \hat{\psi}$ of the previous subsection. If $A$ is an event measurable with respect to these processes, we define

$$
\overline{\mathbb{P}}_{N, r}(A)=\frac{\mathbb{E}_{N, r}\left(\mathbb{I}_{A} \partial \psi_{\varnothing}^{\mathfrak{t}_{1}} \partial \hat{\psi}_{\varnothing}^{\mathfrak{t}_{2}}\right)}{\mathbb{E}_{N, r}\left(\partial \psi_{\varnothing}^{\mathfrak{t}_{1}} \partial \hat{\psi}_{\varnothing}^{\mathrm{t}_{2}}\right)}
$$

In this section we prove two estimates relating to $\overline{\mathbb{P}}_{N, r}$. Before we state these, recall our conventions: if $\beta<\infty$ then we write $r=\beta$ and let $\mathfrak{t}_{1}=\mathfrak{t}_{2}=\mathfrak{p}$, whereas if $\beta=\infty$ then $r=2 N \rightarrow \infty$ and $\mathfrak{t}_{1}=\mathfrak{f}, \mathfrak{t}_{2}=\mathfrak{w}$. In the following result we let $N_{0}<N$ and if $\beta<\infty$ let $r_{0}=r=\beta$, or if $\beta=\infty$ let $r_{0}=2 N_{0}<2 N=r$. If $J$ is a measurable subset of $K(N, r)$ we say that the event $A$ is defined in $J$ if it is measurable with respect to the restrictions of the processes $B, \hat{B}, G, \Delta, \psi, \hat{\psi}$ to $J$.

Proposition 2.2.

(A) For each $(x, t) \in K(N, r)$ there is a constant $C_{(x, t)}$ not depending on $N, r$ such that

$$
\left\langle\sigma_{0,0} \sigma_{x, t}\right\rangle_{N, r}^{\mathfrak{w}, \mathfrak{t}_{2}}-\left\langle\sigma_{0,0} \sigma_{x, t}\right\rangle_{N, r}^{\mathfrak{f}, \mathfrak{t}_{1}} \leq C_{(x, t)} \overline{\mathbb{P}}_{N, r}((0,0) \leftrightarrow \Gamma) .
$$

(B) There is a constant $c=c\left(N_{0}, r_{0}\right)$ such that the following holds. 
Let $A$ be an event defined in $K(N, r) \backslash K\left(N_{0}, r_{0}\right)$, and let $\mathcal{C}$ be the event that there is an open path inside $K\left(N_{0}, r_{0}\right)$ between every pair of points in $K\left(N_{0}, r_{0}\right)$. Then

$$
\overline{\mathbb{P}}_{N, r}(A) \leq c \overline{\mathbb{P}}_{N, r}(A \cap \mathcal{C}) .
$$

In proving Proposition 2.2 we will be using the following fact about 'local modifications' of point processes. Let $X$ denote a point process on the interval $[0, t]$. Let $\tilde{X}$ be another point process on $[0, t]$ obtained from $X$ by a deterministic or random modification. For example, $\tilde{X}$ may be obtained by adding a point somewhere in $[0, t]$, or deleting one of the points of $X$. Write $E, \tilde{E}, \mathbb{E}$ for the law of $X$, the law of $\tilde{X}$ and their joint law, respectively. We will assume that $\tilde{X}$ is defined in such a way that for some event $A$ we have $\tilde{X} \in A$ with probability 1 . Moreover, assume that $f$ is some function and $c_{1}, c_{2}>0$ some constants such that $\frac{f(X)}{f(\tilde{X})} \leq c_{1}$ and the Radon-Nikodym density $\frac{d \tilde{E}}{d E} \leq c_{2}$ almost surely. We then have that

$$
\begin{aligned}
E[f(X)] & =\mathbb{E}[f(X)]=\mathbb{E}[f(X) \mathbb{I}\{\tilde{X} \in A\}] \leq c_{1} \mathbb{E}[f(\tilde{X}) \mathbb{I}\{\tilde{X} \in A\}] \\
& =c_{1} \tilde{E}\left[f(\tilde{X}) \mathbb{I}_{A}(\tilde{X})\right]=c_{1} E\left[\frac{d \tilde{E}}{d E}(X) f(X) \mathbb{I}_{A}(X)\right] \\
& \leq c_{1} c_{2} E[f(X) \mathbb{I}\{X \in A\}] .
\end{aligned}
$$

We will be using (15) when $X$ is a Poisson process of intensity $\alpha$, say, and $\tilde{X}$ is obtained in one of the following three ways.

- Firstly, if $\tilde{X}$ is the trivial process obtained by deleting all points of $X$ then

$$
\frac{d \tilde{E}}{d E}(X)=e^{\alpha t} \mathbb{I}\{X=\varnothing\} \leq e^{\alpha t} .
$$

- Secondly, suppose $\tilde{X}$ is obtained from $X$ by adding two independent, uniformly distributed points if $X=\varnothing$, but letting $\tilde{X}=X$ otherwise. Then

$$
\begin{aligned}
\frac{d \tilde{E}}{d E}(X) & =\mathbb{I}\{X \neq \varnothing\}+\frac{2}{(\alpha t)^{2}} \mathbb{I}\{|X|=2\} \\
& \leq 1+\frac{2}{(\alpha t)^{2}} .
\end{aligned}
$$

- Thirdly, suppose $\tilde{X}$ is obtained from $X$ by adding uniformly a point if $|X| \in\{0,1\}$, alternatively deleting a uniformly chosen point if $|X| \geq 2$. Then

$$
\begin{aligned}
\frac{d \tilde{E}}{d E}(X) & =\frac{1}{\alpha t} \mathbb{I}\{|X|=1\}+\frac{2}{\alpha t} \mathbb{I}\{|X|=2\}+\mathbb{I}\{X \neq \varnothing\} \frac{\alpha t}{|X|+1} \\
& \leq \frac{2}{\alpha t}+\alpha t .
\end{aligned}
$$


One way to check (16)-(18) is to approximate $X$ by a Bernoulli process on $\left\{0, \frac{1}{n}, \ldots, \frac{\lfloor n\rfloor}{n}\right\}$ with success probability $\alpha / n$ and look at the limits of the corresponding likelihood ratios.

Proof of Proposition 2.2. We begin with the easier part (B). The statement is equivalent to

$$
\mathbb{E}_{N, r}\left(\mathbb{I}_{A} \partial \psi_{\varnothing}^{\mathrm{t}_{1}} \partial \hat{\psi}_{\varnothing}^{\mathrm{t}_{2}}\right) \leq c \mathbb{E}_{N, r}\left(\mathbb{1}_{A} \mathbb{I}_{\mathcal{C}} \partial \psi_{\varnothing}^{\mathrm{t}_{1}} \partial \hat{\psi}_{\varnothing}^{\mathrm{t}_{2}}\right) .
$$

We modify $\Delta$ by removing all points in $K\left(N_{0}, r_{0}\right)$ and we modify $B$ inside $K\left(N_{0}, r_{0}\right)$ as in (17). That is, whenever $B_{x y} \cap I_{r_{0}}=\varnothing$ we add two bridges uniformly placed in $I_{r_{0}}$, otherwise leave $B_{x y}$ unchanged. The resulting bridge-configuration $\tilde{B}$ is then still consistent with source set $\varnothing$. If $\beta=\infty$ there is (due to our choice of boundary conditions $\mathfrak{t}_{1}=\mathfrak{f}$, $\left.\mathfrak{t}_{2}=\mathfrak{w}\right)$ a unique labelling $\tilde{\psi}_{\varnothing}^{\mathfrak{t}_{1}}$ consistent with $\tilde{B}$ which agrees with the original labelling $\psi_{\varnothing}^{\mathfrak{t}_{1}}$ in $K(N, r) \backslash K\left(N_{0}, r_{0}\right)$. If $\beta<\infty$ there is a unique labelling $\tilde{\psi}_{\varnothing}^{\mathrm{t}_{1}}$ which agrees with $\psi_{\varnothing}^{\mathrm{t}_{1}}$ in $K(N, r) \backslash K\left(N_{0}, r_{0}\right)$ and at each $(x, 0)$ such that $x \in \Lambda_{N_{0}}$. Since we have removed all cuts and placed bridges between all neighbouring pairs of intervals, the event $\mathcal{C}$ holds after the modification. Since all changes have been restricted to $K\left(N_{0}, r_{0}\right)$ the change preserves the event $A$. The total length $\tilde{\epsilon}$ of intervals labelled 'even' in $\tilde{\psi}_{\varnothing}^{\mathrm{t}_{1}}$ satisfies $\tilde{\epsilon} \geq \epsilon-r_{0}\left(2 N_{0}+1\right)^{d}$. Applying (15) with $f$ equal to the weight of the labelling, as well as (16) and (17), we obtain (19) with

$$
c=\exp \left(4 \delta r_{0}\left(2 N_{0}+1\right)^{d}\right) \exp \left(4 \delta r_{0}\left(2 N_{0}+1\right)^{d}\right)\left(1+2 /\left(\lambda r_{0}\right)^{2}\right)^{2 d\left(2 N_{0}+1\right)^{d}} .
$$

(The first factor is due to the change in the weight of the labelling, the second to the change of measure of $\Delta$, and the third to the change of measure of $B$.)

We now turn to part (A). By (13) it suffices to show that

$$
\mathbb{E}_{N, r}\left(\partial \psi_{\varnothing}^{\mathrm{t}_{1}} \partial \hat{\psi}_{0 \kappa}^{\mathrm{t}_{2}} \mathbb{I}\{0 \leftrightarrow \Gamma\}\right) \leq C_{\kappa} \mathbb{E}_{N, r}\left(\partial \psi_{\varnothing}^{\mathrm{t}_{1}} \partial \hat{\psi}_{\varnothing}^{\mathrm{t}_{2}} \mathbb{I}\{0 \leftrightarrow \Gamma\}\right),
$$

where $\kappa=(x, t)$. We begin with the (more delicate) case when $\beta=\infty$ and $r=2 N$.

For each $y \in \Lambda_{N}$ write

$$
I_{y, k}=\{y\} \times(k, k+1], \quad-N \leq k \leq N-1 .
$$

Thus the $I_{y, k}$ form a partition of $K(N, r)$ into intervals of length 1 , and we have that $(x, t) \in I_{x,[t]-1}$ and $(0,0) \in I_{0,-1}$. We begin by defining a collection $\Pi(x, t)$ of intervals of this form which 'connect' $(0,0)$ to $(x, t)$. There is some flexibility in the choice of $\Pi(x, t)$, but for definiteness we define it as follows. Firstly, let $0=x_{0}, x_{1}, \ldots, x_{n}=x$ be a fixed, shortest nearest-neighbour path from 0 to $x$ in $\Lambda_{N}$; thus $n=\|x\|$. Next, set $k_{0}=-1$ and

$$
\begin{aligned}
& k_{1}=0, k_{2}=1, \ldots, k_{m}=\lceil t\rceil-1 \quad \text { if } t>0, \\
& k_{1}=-2, k_{2}=-3, \ldots, k_{m}=\lceil t\rceil-1 \quad \text { if } t<0 \text {. }
\end{aligned}
$$



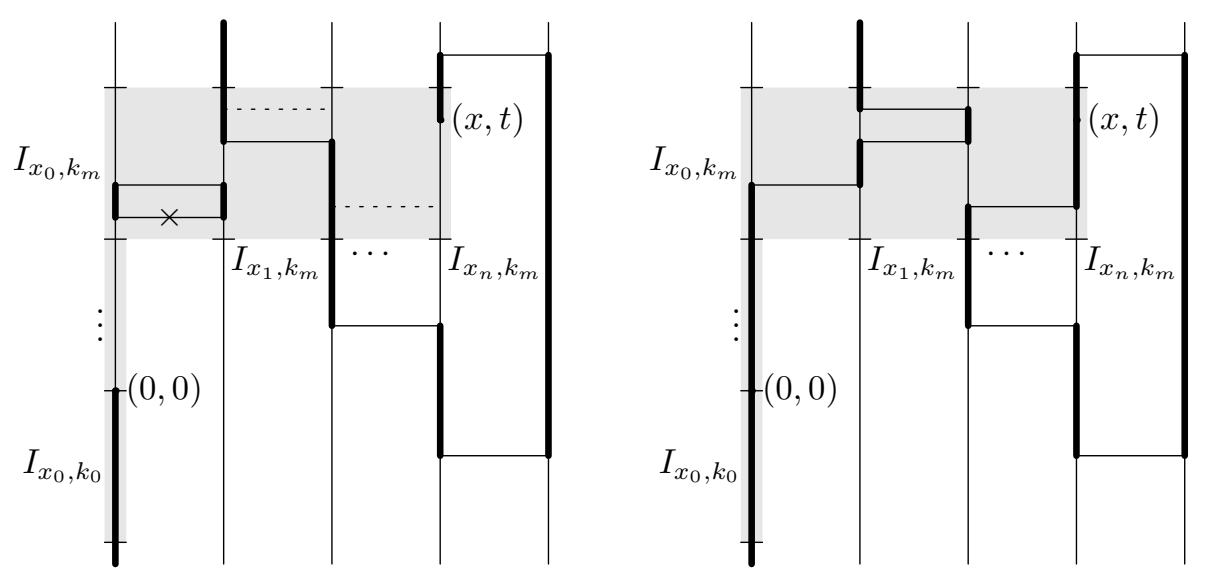

Figure 2. Part of the labellings $\hat{\psi}_{0 \kappa}^{\mathfrak{w}}$ (left) and $\tilde{\psi}_{\varnothing}^{\mathfrak{w}}$ (right) in the proof of Proposition 2.2(A). The intervals comprising $\Pi(\kappa)$ are indicated and highlighted in light grey. Intervals labelled 'odd' are drawn bold. Bridges added (respectively, deleted) are drawn dashed (respectively, marked with an $x$ ).

We define

$$
\Pi(\kappa)=\Pi(x, t)=\left\{I_{0, k_{0}}, I_{0, k_{1}}, \ldots, I_{0, k_{m}}, I_{x_{1}, k_{m}}, I_{x_{2}, k_{m}}, \ldots, I_{x_{n}, k_{m}}\right\} .
$$

Note that $(0,0) \in I_{0, k_{0}}$, that $(x, t) \in I_{x_{n}, k_{m}}$, and that the number of intervals in $\Pi(x, t)$ as well as their total length are bounded by $|t|+2+\|x\|$.

We are going to modify $\Delta, \hat{B}$ and $\hat{\psi}$ along $\Pi(x, t)$ and apply the argument at (15). We modify $\Delta$ by simply replacing $\Delta \cap I$ with $\varnothing$ for all $I \in \Pi(x, t)$. By (16) the corresponding Radon-Nikodym density is at $\operatorname{most} \exp (4 \delta(|t|+2+\|x\|))$. Next, define

$$
J_{i}=\left\{\left(x_{i} x_{i+1}, s\right):\left(x_{i}, s\right) \in I_{x_{i}, k_{m}},\left(x_{i+1}, s\right) \in I_{x_{i+1}, k_{m}}\right\} .
$$

We modify $\hat{B}$ by applying the operation in (18) in each $J_{i}$ for $0 \leq$ $i \leq n-1$; that is, if $J_{i}$ contains 0 or 1 bridge we add one uniformly, but if $J_{i}$ contains 2 or more bridges we delete one chosen uniformly. Write $\tilde{B}$ for the modified process of bridges. By (18), the corresponding Radon-Nikodym density is at most $(2 / \lambda+\lambda)^{|t|+2+\|x\|}$.

If $\hat{B}$ was consistent with the sources $0, \kappa$ then $\tilde{B}$ is consistent with the source set $\varnothing$. Due to the boundary condition there is a unique labeling $\tilde{\psi}_{\varnothing}^{\mathfrak{w}}$ associated with $\tilde{B}$, and in fact one obtains $\tilde{\psi}_{\varnothing}^{\mathfrak{w}}$ from $\hat{\psi}_{0 \kappa}^{\mathfrak{w}}$ by modifying the labels in intervals belonging to $\Pi(x, t)$ only. See Figure 2 . It follows that

$$
\frac{\partial \hat{\psi}_{0 \kappa}^{\mathfrak{w}}}{\partial \tilde{\psi}_{\varnothing}^{\mathfrak{w}}} \leq \exp (2 \delta(|t|+2+\|x\|))
$$


Note that the modifications described above do not destroy any connections (but possibly creates some new ones). In particular, if $0 \leftrightarrow \Gamma$ before then also $0 \leftrightarrow \Gamma$ after. Applying the argument in (15) we therefore arrive at (20), with

$$
C_{(x, t)}=\exp (6 \delta(|t|+2+\|x\|))(2 / \lambda+\lambda)^{|t|+2+\|x\|} .
$$

We now turn to the case $\beta<\infty$, and recall that we then have $r=\beta$. We no longer need to partition $I_{\beta}$ into intervals of length 1 , but instead define $I_{x}=\{x\} \times I_{\beta}$. We now let

$$
\Pi(x, t)=\left\{I_{x_{0}}, I_{x_{1}}, I_{x_{2}}, \ldots, I_{x_{n}}\right\},
$$

where as before $0=x_{0}, x_{1}, \ldots, x_{n}=x$ is a fixed, shortest, nearestneighbour path from 0 to $x$ in $\Lambda_{N}$. The number of intervals in $\Pi(x, t)$ is now $n=\|x\|$, and their combined length is $\beta\|x\|$. With this definition of $\Pi(x, t)$ we apply the same modifications to $\Delta$ and $\hat{B}$ as in the case $\beta=\infty$. Now we let $\tilde{\psi}_{\varnothing}^{\mathfrak{p}}$ be the unique labelling which agrees with $\hat{\psi}_{0 \kappa}^{\mathfrak{p}}$ outside the intervals of $\Pi(x, t)$ and at all points $\left(x_{i}, 0\right)$ for $i=0,1, \ldots, n$. This time we get (20) with

$$
C_{(x, t)}=\exp (6 \delta \beta\|x\|)(2 / \lambda \beta+\lambda \beta)^{\|x\|} .
$$

\section{Infinite-VOLUME RPR}

In this section we study the limit $\overline{\mathbb{P}}$ of the measures $\overline{\mathbb{P}}_{N, r}$ as $N \rightarrow \infty$ (and either $r=\beta<\infty$ is fixed, or $r=2 N \rightarrow \infty$ ). In Section 3.1 we prove the existence of $\overline{\mathbb{P}}$ as well as basic properties such as translationinvariance and ergodicity. Then in Section 3.2 we show how the argument of Burton and Keane [11] can be adapted to show that, almost surely under $\overline{\mathbb{P}}$, there is either no or exactly one infinite connected cluster.

3.1. Existence and basic properties. In proving existence of the limit $\overline{\mathbb{P}}$ of the sequence $\overline{\mathbb{P}}_{N, r}$ we will need to pay attention to the underlying point processes, and we will loosely follow the notational conventions of Daley and Vere-Jones [14] for point processes. Recall that the labelling $\psi_{\varnothing}^{\mathfrak{t}_{1}}$ is a function of the pair $(B, \tau)$ where $B$ is a point process on $\mathcal{E}_{N} \times I_{r}$ and $\tau \in\{0,1\}^{\Lambda_{N}}$, and similarly $\hat{\psi}_{\varnothing}^{\mathrm{t}_{2}}$ is a function of $(\hat{B}, \hat{G}, \hat{\tau})$. (If $\beta=\infty$ then $\tau, \hat{\tau}$ are redundant due to the boundary conditions.) Write

$$
\mathcal{X}=\mathcal{X}_{\beta}=\left[\bigcup_{i=0}^{d}\left(\mathbb{Z}^{d}+\frac{1}{2} e_{i}\right)\right] \times I_{\beta}, \quad T=\{0,1\}^{\mathbb{Z}^{d}},
$$

where $e_{0}$ is the zero vector and, for $i \neq 0, e_{i}$ is the unit vector in the $i$ :th coordinate. Writing $\mathcal{N}=\mathcal{N}_{\mathcal{X}}$ for the set of boundedly finite point processes (counting measures) on $\mathcal{X}$ (denoted $\mathcal{N}_{\mathcal{X}}^{\#}$ in [14]), an obvious 
mapping allows us to see both $B$ and $\hat{B} \cup \hat{G}$ as random elements of $\mathcal{N}$. The measure $\overline{\mathbb{P}}_{N, r}$ factorizes as

$$
\overline{\mathbb{P}}_{N, r}=\mathbb{P}_{N, r}^{\mathrm{t}_{1}} \times \hat{\mathbb{P}}_{N, r}^{t_{2}} \times P_{\Delta},
$$

where $P_{\Delta}$ is the law of $\Delta$ and $\mathbb{P}_{N, r}^{\mathrm{t}_{1}}, \hat{\mathbb{P}}_{N, r}^{\mathrm{t}_{2}}$ are the measures on $\mathcal{N} \times T$ governing $(B, \tau)$ and $(\hat{B} \cup \hat{G}, \hat{\tau})$ respectively, given by

$$
\frac{d \mathbb{P}_{N, r}^{t_{1}}}{d E_{N, r}}=\frac{\partial \psi_{\varnothing}^{t_{1}}}{E_{N, r}\left(\partial \psi_{\varnothing}^{t_{1}}\right)}, \quad \frac{d \hat{\mathbb{P}}_{N, r}^{t_{2}}}{d \hat{E}_{N, r}}=\frac{\partial \hat{\psi}_{\varnothing}^{t_{2}}}{\hat{E}_{N, r}\left(\partial \hat{\psi}_{\varnothing}^{t_{2}}\right)} .
$$

Write $\Omega=(\mathcal{N} \times T)^{2} \times \mathcal{N}$ and note that $\mathcal{N}$, and hence also $\Omega$, is a complete and separable metric space [14, Proposition 9.1.IV]. We equip $\Omega$ with the Borel $\sigma$-algebra $\mathcal{F}$, which coincides with the $\sigma$-algebra generated by finite-dimensional distributions. Recall that $r$ is either fixed (if $\beta<\infty$ ) or $r=2 N$ (if $\beta=\infty$ ).

Proposition 3.1. The measures $\overline{\mathbb{P}}_{N, r}$ converge weakly to a probability measure $\overline{\mathbb{P}}$ on $\Omega$ as $N \rightarrow \infty$.

Proof. The measure $P_{\Delta}$ does not depend on $N$, so by [ 6 , Theorem 2.8] convergence of $\overline{\mathbb{P}}_{N, r}$ follows once we show convergence of $\mathbb{P}_{N, r}^{t_{1}}$ and $\hat{\mathbb{P}}_{N, r}^{t_{2}}$. We give full details for the case of $\mathbb{P}_{N, r}^{t_{1}}$, the case of $\hat{\mathbb{P}}_{N, r}^{t_{2}}$ is similar. We first show that the sequence $\mathbb{P}_{N, r}^{t_{1}}$ is tight, i.e. every subsequence can be refined to a further subsequence along which $\mathbb{P}_{N, r}^{t_{1}}$ converges. We then show that there is a $\pi$-system $\mathcal{A}_{0}$ such that the limit of $\mathbb{P}_{N, r}^{\mathrm{t}_{1}}(A)$ exists for each $A \in \mathcal{A}_{0}$. The result then follows in the standard way from Prohorov's theorem.

Turning to the tightness of $\mathbb{P}_{N, r}^{t_{1}}$, note that since $T$ is compact it suffices to show that the marginal of $\mathbb{P}_{N, r}^{t_{1}}$ on $\mathcal{N}$ is tight. A criterion for this is given in [14, Proposition 11.1.VI]. Fix $N_{0}, r_{0}$ and write $X=\mid B \cap$ $F\left(N_{0}, r_{0}\right) \mid$ for the number of points of $B$ 'inside' $K\left(N_{0}, r_{0}\right)$. Tightness follows if we show that for for each $\varepsilon>0$ there is $m$ such that

$$
\mathbb{P}_{N, r}^{t_{1}}(X>m)<\varepsilon \text { for all } N, r .
$$

By Markov's inequality it suffices to show that there is a constant $C\left(N_{0}, r_{0}\right)$ not depending on $N, r$ such that the expectation

$$
\mathbb{P}_{N, r}^{t_{1}}[X] \leq C\left(N_{0}, r_{0}\right) \text { for all } N, r .
$$

In proving (22) we will require the following notation. Write $K=$ $K(N, r), K_{0}=K\left(N_{0}, r_{0}\right), K^{\prime}=K \backslash K_{0}, F=F(N, r), F_{0}=F\left(N_{0}, r_{0}\right)$, $F^{\prime}=F \backslash F_{0}, B^{\prime}=B \cap F^{\prime}$, and $B^{(0)}=B \cap F_{0}$. For briefer notation write $\psi$ for the labelling $\psi_{\varnothing}^{t_{1}}$, and let $\psi^{\prime}$ denote the restriction of $\psi$ to $K^{\prime}$. Recall that $\epsilon$ denotes the total Lebesgue measure of $K$ labelled 'even' in $\psi$, and write $\epsilon^{\prime}$ for the Lebesgue measure of the 'even' subset of $K^{\prime}$. Letting $\mathcal{C}$ denote the event that $B$ is consistent (with source 
set $\varnothing)$ we have that $\partial \psi=e^{2 \delta \epsilon} \mathbb{I}_{\mathcal{C}}$. Clearly $\epsilon^{\prime} \leq \epsilon \leq \epsilon^{\prime}+\left|K_{0}\right|$, where $\left|K_{0}\right|=\left(2 N_{0}+1\right)^{d} r_{0}$ denotes the total Lebesgue measure of $K_{0}$, and it follows that

$$
\mathbb{P}_{N, r}^{\mathfrak{t}_{1}}[X] \leq e^{2 \delta\left|K_{0}\right|} \frac{E_{N, r}\left(X e^{2 \delta \epsilon^{\prime}} \mathbb{I}_{\mathcal{C}}\right)}{E_{N, r}\left(e^{2 \delta \epsilon^{\prime}} \mathbb{I}_{\mathcal{C}}\right)}
$$

The difficulty lies in the fact that although $X$ is a function of $B^{(0)}$ only, both $\epsilon^{\prime}$ and $\mathcal{C}$ depend both on both $B^{(0)}$ and $B^{\prime}$. To 'separate' this dependence, we introduce the notation

$Z_{x y}=\left|B_{x y} \cap I_{r}\right|, \quad Z_{x y}^{(0)}=\left|B_{x y} \cap I_{r_{0}}\right|, \quad Z_{x y}^{\prime}=\left|B_{x y} \backslash I_{r_{0}}\right|, \quad\left(x y \in \mathcal{E}_{N}\right)$.

Thus $Z_{x y}=Z_{x y}^{(0)}+Z_{x y}^{\prime}$, and the $Z_{x y}^{(0)}, Z_{x y}^{\prime}$ are independent Poisson random variables under $E_{N, r}$ (with parameters $\lambda r_{0}$ and $\lambda\left(r-r_{0}\right)$, respectively). The number of switching points on $\{x\} \times I_{r}$ can be written

$$
\left|S_{x}\right|=\sum_{\substack{y \in \Lambda_{N} \\ y \sim x}} Z_{x y}
$$

and letting $S_{x}^{(0)}$ denote the set of switching points in $\{x\} \times I_{r_{0}}$ we can similarly write

$$
\left|S_{x}^{(0)}\right|=\sum_{\substack{y \in \Lambda_{N_{0}} \\ y \sim x}} Z_{x y}^{(0)} .
$$

The process $B^{\prime}$ imposes parity constraints on $B^{(0)}$ which can be described in terms of the random vector $\pi=\left(\pi_{x}: x \in \Lambda_{N_{0}}\right) \in\{0,1\}^{\Lambda_{N_{0}}}$ given by

$$
\pi_{x} \equiv \sum_{\substack{y \notin \Lambda_{N_{0}} \\ y \sim x}} Z_{x y}^{(0)}+\sum_{\substack{y \in \Lambda_{N} \\ y \sim x}} Z_{x y}^{\prime} .
$$

(Here and in what follows we write $\equiv$ for congruence modulo 2.) Note that $\pi$ is a function of $B^{\prime}$ only, and that $\mathcal{C}=\mathcal{C}^{\prime} \cap \mathcal{C}^{(0)} \cap \tilde{\mathcal{C}}$, where

$$
\begin{aligned}
\mathcal{C}^{\prime} & =\left\{\left|S_{x}\right| \equiv 0 \forall x \in \Lambda_{N} \backslash \Lambda_{N_{0}}\right\}, \\
\mathcal{C}^{(0)} & =\left\{\left|S_{x}^{(0)}\right| \equiv \pi_{x} \forall x \in \Lambda_{N_{0}}\right\}, \\
\tilde{\mathcal{C}} & =\left\{\exists z \in\{0,1\}^{\mathcal{E}_{N_{0}}}: \forall x \in \Lambda_{N_{0}}, \sum_{\substack{y \sim x \\
y \in \Lambda_{N_{0}}}} z_{x y} \equiv \pi_{x}\right\} .
\end{aligned}
$$

Strictly speaking the event $\tilde{\mathcal{C}}$ is redundant as it is implied by $\mathcal{C}^{(0)}$, however it is useful to keep since it, in contrast to $\mathcal{C}^{(0)}$, depends on $B^{\prime}$ only. For each realization of $\pi$ such that $\tilde{\mathcal{C}}$ holds we fix a deterministic vector $z$ as in the definition of $\tilde{\mathcal{C}}$. Note that the number of possible $\pi$ is at most $2^{\left|\Lambda_{N_{0}}\right|}$.

In the numerator of (23) we have

$$
\begin{aligned}
E_{N, r}\left(X e^{2 \delta \epsilon^{\prime}} \mathbb{1}_{\mathcal{C}}\right) & =E_{N, r}\left(e^{2 \delta \epsilon^{\prime}} \mathbb{1}_{\mathcal{C}^{\prime} \cap \tilde{\mathcal{C}}} E_{N, r}\left(X \mathbb{1}_{\mathcal{C}^{(0)}} \mid \psi^{\prime}\right)\right) \\
& \leq E_{N, r}(X) E_{N, r}\left(e^{2 \delta \epsilon^{\prime}} \mathbb{1}_{\mathcal{C}^{\prime} \cap \tilde{\mathcal{C}}}\right)
\end{aligned}
$$


where we bounded $\mathbb{1}_{\mathcal{C}^{(0)}}$ by 1 and used the fact that $X$ is independent of $\psi^{\prime}$. Note that $E_{N, r}(X)=\lambda r_{0}\left|\mathcal{E}_{N_{0}}\right|$. In the denominator of (23) we have

$$
E_{N, r}\left(e^{2 \delta \epsilon^{\prime}} \mathbb{1}_{\mathcal{C}}\right)=E_{N, r}\left(e^{2 \delta \epsilon^{\prime}} \mathbb{1}_{\mathcal{C}^{\prime} \cap \tilde{\mathcal{C}}} P_{N, r}\left(\mathcal{C}^{(0)} \mid \psi^{\prime}\right)\right),
$$

which we need to bound from below. We claim that there is an $\varepsilon=$ $\varepsilon\left(N_{0}, r_{0}\right)>0$ such that $P_{N, r}\left(\mathcal{C}^{(0)} \mid \psi^{\prime}\right) \geq \varepsilon$ for all realizations $\psi^{\prime}$ such that $\tilde{\mathcal{C}}$ holds. Indeed, recall that we fixed a deterministic vector $z$ for each $\pi$ such that $\tilde{\mathcal{C}}$ holds. The event $\tilde{\mathcal{C}}_{z}=\left\{Z_{x y}^{(0)}=z_{x y} \forall x y \in \mathcal{E}_{N_{0}}\right\}$ thus implies $\mathcal{C}^{(0)}$. Under $P_{N, r}\left(\cdot \mid \psi^{\prime}\right)$ the $Z_{x y}^{(0)}$ are independent Poisson random variables, so each $\tilde{\mathcal{C}}_{z}$ has positive probability. The claim thus holds with $\varepsilon$ being the minimum of $P_{N, r}\left(\tilde{\mathcal{C}}_{z} \mid \psi^{\prime}\right)$ over the (at most $2^{\left|\Lambda_{N_{0}}\right|}$ ) choices of $z$. Therefore (22) follows with

$$
C\left(N_{0}, r_{0}\right)=e^{2 \delta\left|K_{0}\right|} \lambda r_{0}\left|\mathcal{E}_{N_{0}}\right| / \varepsilon\left(N_{0}, r_{0}\right) .
$$

Having proved tightness of the sequence $\mathbb{P}_{N, r}^{t_{1}}$ we now turn to showing uniqueness of subsequential limits. Let $\mathcal{A}_{0}$ denote the collection of events of the form

$$
A=\{\psi \text { is 'even' in } J\},
$$

where $J$ is any finite union of bounded closed intervals in $\mathbb{K}_{\beta}$. (We allow intervals of length 0 , i.e. isolated points.) Then $\mathcal{A}_{0}$ is a $\pi$-system which generates the $\sigma$-algebra $\mathcal{F}$ (note that the process $B$ can be recovered from the labelling $\psi$ ). We let $N$ be large enough that $J \subseteq K(N, r)$. By (11) and [9, Lemma 3.2] we have

$$
\mathbb{P}_{N, r}^{\mathfrak{t}_{1}}(A)=c(J) \mu_{N, r}^{\mathfrak{f}, \mathfrak{t}_{1}}\left[\exp \left(-\lambda L_{J}(\sigma)\right)\right],
$$

for some constant $c(J)$ depending only on $J$, and where

$$
L_{J}(\sigma)=\sum_{x y \in \mathcal{E}_{N}} \int_{I_{r}} \sigma(x, t) \sigma(y, t) \mathbb{I}\{(x y, t) \in \tilde{J}\} d t
$$

and $\tilde{J}$ is the set of point $(y z, t) \in \mathbb{F}$ such that $(y, t) \in J$ or $(z, t) \in J$ (or both). By [7, Theorem 2.5.1] the sequence of measures $\mu_{N, r}^{\mathfrak{f}, \mathfrak{t}_{1}}$ converges weakly, hence the probability in (24) converges.

For later reference we note that the constant $c(J)$ in (24) can be written as

$$
c(J)=2^{-n} e^{\delta|J|+\lambda|\tilde{J}|} .
$$

Here $|J|$ and $|\tilde{J}|$ denote the total length of the intervals comprising $J$ and $\tilde{J}$, and $n$ is the difference between the number of intervals comprising $K^{\prime}=K \backslash J$ and the number of intervals comprising $K$ (in counting the number of intervals we view $I_{\beta}$ as a circle when $\left.\mathfrak{t}=\mathfrak{p}\right)$.

For $(x, s) \in \mathbb{K}_{\beta}$ define the translation or shift $\tau_{(x, s)}: \mathbb{K}_{\beta} \rightarrow \mathbb{K}_{\beta}$ by $\tau_{(x, s)}(y, t)=(y+x, t+s)$ where in the case $\beta<\infty$ we view $t+s$ modulo $\beta$. For a function $\zeta: \mathbb{K}_{\beta} \rightarrow \mathbb{R}$ (e.g. a labelling $\psi$ or a spin-configuration 
$\sigma)$ we define $\tau_{(x, s)}(\zeta)$ by $\left[\tau_{(x, s)}(\zeta)\right](y, t)=\zeta(y+x, t+s)$. We write $\tau_{x}$ for $\tau_{(x, 0)}$.

Proposition 3.2. The measure $\overline{\mathbb{P}}$ is invariant with respect to the shifts $\tau_{(x, s)}$, and ergodic with respect to the shifts $\tau_{x}$ for $x \neq 0$.

Proof. We use the decomposition (21). The measure $P_{\Delta}$ is translationinvariant and ergodic, so it suffices to show that the weak limits of $\mathbb{P}_{N, r}^{t_{1}}$ and $\hat{\mathbb{P}}_{N, r}^{t_{2}}$ and are translation-invariant and ergodic. Again, we give details for $\mathbb{P}_{N, r}^{t_{1}}$. Let $A \in \mathcal{A}_{0}$, let $(x, s) \in \mathbb{K}_{\beta}$, and let $N$ be large enough that $\tau_{(x, s)}^{-1} J \subseteq K(N, r)$ (recall that $r=2 N$ if $\beta=\infty$ and $r=\beta$ if $\beta<\infty)$. We have as in (24) that

$$
\mathbb{P}_{N, r}^{\mathfrak{t}_{1}}\left(\tau_{(x, s)} A\right)=c\left(\tau_{(x, s)}^{-1} J\right) \mu_{N, r}^{\mathfrak{f}, \mathfrak{t}_{1}}\left[\exp \left(-\lambda L_{\tau_{(x, s)}^{-1} J}(\sigma)\right)\right] .
$$

From (25) we see that $c\left(\tau_{(x, s)}^{-1} J\right)=c(J)$. By [7, Theorem 2.5.1],

$$
\begin{aligned}
\lim _{N \rightarrow \infty} \mu_{N, r}^{\mathfrak{f}, \mathfrak{t}_{1}}\left[\exp \left(-\lambda L_{\tau_{(x, s)}^{-1} J}(\sigma)\right)\right] & =\lim _{N \rightarrow \infty} \mu_{N, r}^{\mathfrak{f}, \mathfrak{t}_{1}}\left[\exp \left(-\lambda L_{J}(\sigma)\right)\right] \\
& =\mu^{\left(\mathfrak{f}, \mathfrak{t}_{1}\right)}\left[\exp \left(-\lambda L_{J}(\sigma)\right)\right],
\end{aligned}
$$

and hence $\lim _{N \rightarrow \infty} \mathbb{P}_{N, r}^{\mathrm{t}_{1}}\left(\tau_{(x, s)} A\right)=\lim _{N \rightarrow \infty} \mathbb{P}_{N, r}^{\mathrm{t}_{1}}(A)$. This proves translationinvariance on the $\pi$-system $\mathcal{A}_{0}$, which by the $\pi$-systems lemma implies full translation-invariance.

Let $A_{1}, A_{2} \in \mathcal{A}_{0}$ be the events that $\psi$ is 'even' in $J_{1}$ and $J_{2}$, respectively. For $\|x\|$ and $N$ large enough we have from (24) and (25) that

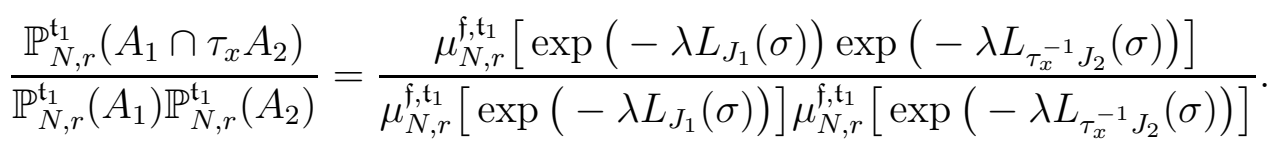

Letting $N \rightarrow \infty$ and writing $\mathbb{P}^{\left(\mathfrak{t}_{1}\right)}$ for the weak limit of $\mathbb{P}_{N, r}^{\mathfrak{t}_{1}}$ it follows that

$$
\frac{\mathbb{P}^{\left(\mathfrak{t}_{1}\right)}\left(A_{1} \cap \tau_{x} A_{2}\right)}{\mathbb{P}^{\left(\mathfrak{t}_{1}\right)}\left(A_{1}\right) \mathbb{P}^{\left(\mathfrak{t}_{1}\right)}\left(A_{2}\right)}=\frac{\mu^{\left(\mathfrak{f}, \mathfrak{t}_{1}\right)}\left[\exp \left(-\lambda L_{J_{1}}(\sigma)\right) \exp \left(-\lambda L_{J_{2}}\left(\tau_{x}^{-1} \sigma\right)\right)\right]}{\mu^{\left(\mathfrak{f}, \mathfrak{t}_{1}\right)}\left[\exp \left(-\lambda L_{J_{1}}(\sigma)\right)\right] \mu^{\left(\mathfrak{f}, \mathfrak{t}_{1}\right)}\left[\exp \left(-\lambda L_{J_{2}}(\sigma)\right)\right]}
$$

It follows from Lemma 5.1 in the Appendix that the ratio on the righthand-side converges to 1 as $\|x\| \rightarrow \infty$. Thus $\mathbb{P}^{\left(\mathfrak{t}_{1}\right)}$ is mixing on $\mathcal{A}_{0}$, hence also mixing on $\mathcal{F}$ and hence ergodic.

3.2. Percolation. The notions of paths and connectivity, defined for $\overline{\mathbb{P}}_{N, r}$, extend to $\overline{\mathbb{P}}$. Thus $\mathbb{K}_{\beta}$ decomposes into a random collection of connected components or clusters (each of these is a union of intervals bounded by certain elements of $\Delta$ ). Let $U$ denote the number of these clusters which are unbounded. The random variable $U$ (which may be infinite) is invariant under all translations $\tau_{x}$, and hence by

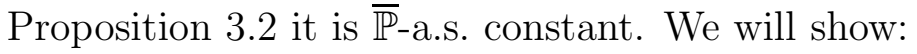

Proposition 3.3. Either $\overline{\mathbb{P}}(U=0)=1$ or $\overline{\mathbb{P}}(U=1)=1$. 
Proof. As this type of argument is fairly standard in percolation theory, and most of the details are the same as for the classical model [2], we only sketch the proof and highlight what adjustments are needed for the quantum case. We focus on the case $\beta=\infty$.

Let $k$ be such that $\overline{\mathbb{P}}(U=k)=1$, we must show that $k \leq 1$. The possibility that $2 \leq k<\infty$ may be ruled out using Proposition 2.2. Roughly speaking, a large enough box $K(N, r)$ will intersect all $k$ unbounded components with positive probability. Using part (B) of Proposition 2.2 one may deduce that, with positive probability, all these components are in fact connected inside $K(N, r)$, a contradiction.

Now assume that $k=\infty$. To get a contradiction one considers what are called coarse-trifurcations. These are points $(x, t) \in \mathbb{K}$ with $x \in\left(2 N_{0}+1\right) \mathbb{Z}^{d}$ and $t \in 2 r_{0} \mathbb{Z}$, having the properties that (i) all points in $K_{0}+(x, t)$ are connected in $K_{0}+(x, t)$, and (ii) $\mathbb{K} \backslash\left(K_{0}+(x, t)\right)$ contains at least 3 distinct unbounded connected components. Here $N_{0}$ and $r_{0}$ are fixed, and may (using Proposition 2.2(B) again) be chosen such that each $(x, t)$ is a coarse-trifurcation with probability $p>0$. Note that $p$ is the same for all $(x, t)$ by translation-invariance. As in [2] one may construct a graph $F$ which reflects the connectivity structure of the coarse-trifurcations in some large box $K(N, r)$. Roughly speaking, the edges of $F$ either connect distinct coarse-trifurcations, or they connect a coarse-trifurcation with an 'element' on the boundary of $K(N, r)$. The latter occurs if the boundary can be reached without having to go too close to another coarse-trifurcation, otherwise the former occurs.

The main difference to [2] is the correct notion of 'element' on the boundary. In their case, where the underlying graphical structure is discrete, one may take the 'elements' simply as vertices on the boundary. In the present case we instead take it to mean a maximal $\Delta$-free interval intersecting the boundary of $K(N, r)$; that is an interval of the form $\{y\} \times I$ with $I \subseteq I_{r}$ maximal such that $\Delta_{y} \cap I=\varnothing$, and such that either $y \in \partial \Lambda_{N}$, or one of the endpoints of $I$ is $\pm r / 2$. With this convention the graph $F$ contains no cycles, as if it did contain a cycle this would violate the definition of coarse-trifurcation. (The important point is that the collection of maximal $\Delta$-free intervals refines the collection of connected components.) Also, the coarse-trifurcations correspond to vertices of $F$ of degree at least 3 . This implies that the number of coarse-trifurcations is at most the number of leaves of $F$, which is in turn bounded above by the number of maximal $\Delta$ free interval intersecting the boundary of $K(N, r)$. The expectation of the latter is easily seen to be at most $2(2 N+1)^{d}+4 \delta r(2 N+1)^{d-1}$, whereas the expected number of coarse-trifurcations is of the order $p\left[r(2 N+1)^{d}\right] /\left[r_{0}\left(2 N_{0}+1\right)^{d}\right]$. Letting $N, r \rightarrow \infty$ this contradicts $p>0$, finishing the proof. 


\section{Proof of the main Result}

4.1. The infrared bound. We now describe the infrared bound of [8] and use it to prove a result of key importance for Theorem 1.1. For technical reasons we will in this section redefine the box $\Lambda_{n}$ as $\{-n+$ $1, \ldots, n\}^{d}$ so that it has even sidelength rather than odd. Recall the Schwinger function (4) and its probabilistic representation (17). Write

$$
c_{N, r}(x, t)=\langle\sigma(0,0) \sigma(x, t)\rangle_{N, r}^{\mathfrak{p}, \mathfrak{p}} .
$$

Note that we use periodic boundary conditions in both directions. Although $c_{N, r}(x, t)$ is defined for $(x, t) \in K(N, r)$, we extend the definition to all of $\mathbb{K}$ by periodicity. We write

$$
\Lambda_{N}^{\star}=\frac{\pi}{N} \Lambda_{N}, \quad I_{r}^{\star}=\frac{2 \pi}{r} \mathbb{Z}, \quad K_{N, r}^{\star}=\Lambda_{N}^{\star} \times I_{r}^{\star} .
$$

Elements of $K_{N, r}^{\star}$ will be denoted $\xi=(k, \ell)$ where $k \in \Lambda_{N}^{\star}$ and $\ell \in I_{r}^{\star}$. For large $N, r$ we may see $\Lambda_{N}^{\star}$ as an approximation of $(-\pi, \pi]^{d}$ and $I_{r}^{\star}$ as an approximation of $\mathbb{R}$. For $p=\left(p_{1}, \ldots, p_{d}\right) \in(-\pi, \pi]^{d}$ let $\hat{L}(p)=\sum_{j=1}^{d}\left(1-\cos \left(p_{j}\right)\right)$ denote the Fourier transform of the graph Laplacian of $\mathbb{Z}^{d}$, and define

$$
E_{\lambda, \delta}(p, q)=\frac{2 \lambda \hat{L}(p)+q^{2} / 2 \delta}{48}, \quad p \in(-\pi, \pi]^{d}, q \in \mathbb{R} .
$$

The Fourier transform of $c_{N, r}$ is

$$
\hat{c}_{N, r}(\xi)=\sum_{x \in \Lambda_{N}} \int_{I_{r}} c_{N, r}(x, t) e^{i k \cdot x} e^{i \ell t} d t, \quad \xi=(k, \ell) \in K_{N, r}^{\star},
$$

where $k \cdot x$ denotes the usual scalar product. Note that $\hat{c}_{N, r}(\xi) \geq 0$. The infrared bound of [8] states that

$$
\text { if } \xi \in K_{N, r}^{\star} \backslash\{0\} \text { then } \hat{c}_{N, r}(\xi) \leq \frac{1}{E_{\lambda, \delta}(\xi)} .
$$

We will use this to show the following:

Lemma 4.1. Suppose $\beta<\infty$ and $d \geq 3$. Then

$$
\lim _{n \rightarrow \infty} \frac{1}{\left|\Lambda_{n}\right|} \sum_{x \in \Lambda_{n}} \int_{I_{\beta}}\langle\sigma(0,0) \sigma(x, t)\rangle_{\lambda_{c}, \beta}^{(\mathfrak{f}, \mathfrak{p})} d t=0 .
$$

Suppose $\beta=\infty$ and $d \geq 2$. Then

$$
\lim _{n, r \rightarrow \infty} \frac{1}{\left|\Lambda_{n}\right| r} \sum_{x \in \Lambda_{n}} \int_{I_{r}}\langle\sigma(0,0) \sigma(x, t)\rangle_{\lambda_{\mathrm{c}}, \infty}^{(\mathfrak{f}, \mathfrak{f})} d t=0 .
$$

Proof. We begin by showing that the stated conditions on $d$ imply the following:

$$
\text { if } \beta<\infty \text { then } \int_{(-\pi, \pi]^{d}} d p \sum_{\ell \in I_{\beta}^{\star}} \frac{1}{E_{\lambda, \delta}(p, \ell)}<\infty,
$$


and

$$
\text { if } \beta=\infty \text { then } \int_{(-\pi, \pi]^{d}} d p \int_{\mathbb{R}} d q \frac{1}{E_{\lambda, \delta}(p, q)}<\infty .
$$

Firstly, in the case $\beta<\infty$ we have that

$$
\begin{aligned}
\int_{(-\pi, \pi]^{d}} d p \sum_{\ell \in I_{\beta}^{\star}} \frac{1}{E_{\lambda, \delta}(p, \ell)} & =\int_{(-\pi, \pi]^{d}} d p \sum_{\ell \in I_{\beta}^{\star}} \frac{48}{2 \lambda \hat{L}(k)+\ell^{2} / 2 \delta} \\
& =\frac{96 \delta}{(2 \pi / \beta)^{2}} \int_{(-\pi, \pi]^{d}} d p \sum_{m \in \mathbb{Z}} \frac{1}{a(p)+m^{2}} \\
& \leq \frac{96 \delta}{(2 \pi / \beta)^{2}} \int_{(-\pi, \pi]^{d}}\left(\frac{1}{a(p)}+\frac{\pi}{a(p)^{1 / 2}}\right) d p
\end{aligned}
$$

where $a(p)=\lambda \delta \beta^{2} \hat{L}(p) / \pi^{2}$. Note that $1 / \hat{L}(p)$ diverges for $p \rightarrow 0$, in the manner of $1 /\|p\|_{2}^{2}$, and that for $\alpha>0$

$$
\int_{(-\pi, \pi]^{d}} \frac{1}{\hat{L}(p)^{\alpha}} d p<\infty \text { if and only if } d>2 \alpha .
$$

Thus (29) holds for $d>2$, i.e. for $d \geq 3$ as claimed. In the case $\beta=\infty$ we have

$$
\begin{aligned}
\int_{(-\pi, \pi]^{d}} d p \int_{-\infty}^{\infty} d q \frac{1}{E_{\lambda, \delta}(p, q)} & =96 \delta \int_{(-\pi, \pi]^{d}} d p \int_{-\infty}^{\infty} d q \frac{1}{4 \lambda \delta \hat{L}(p)+q^{2}} \\
& =48 \pi \sqrt{\delta / \lambda} \int_{(-\pi, \pi]^{d}} \frac{1}{\hat{L}(p)^{1 / 2}} d p .
\end{aligned}
$$

Thus by (31) we have (30) for $d>1$, i.e. for $d \geq 2$ as claimed.

Now define the function

$$
G_{N, r}((x, s),(y, t))=\frac{1}{(2 N)^{d} r} \sum_{(k, \ell) \in K_{N}^{\star} \backslash\{0\}} \frac{e^{-i k \cdot(x-y)} e^{-i \ell(s-t)}}{E_{\lambda, \delta}(k, \ell)},
$$

where $x, y \in \mathbb{Z}^{d}$ and $s, t \in \mathbb{R}$. In the case when $\beta<\infty$ then by Riemann approximation

$$
\begin{aligned}
G_{N, \beta}((x, s),(y, t)) & \rightarrow \frac{1}{(2 \pi)^{d} \beta} \int_{(-\pi, \pi]^{d}} d p \sum_{\ell \in I_{\beta}^{\star}} \frac{e^{-i p \cdot(x-y)} e^{-i \ell(s-t)}}{E_{\lambda, \delta}(p, \ell)} \\
& =: G_{\beta}((x, s),(y, t)), \text { as } N \rightarrow \infty .
\end{aligned}
$$

From (29) we deduce that

$$
\frac{1}{(2 n)^{d}} \sum_{x \in \Lambda_{n}} \int_{I_{\beta}} d t G_{\beta}((0,0),(x, t)) \rightarrow 0 \text { as } n \rightarrow \infty,
$$

which in turn implies that

$$
\frac{1}{(2 n)^{2 d}} \sum_{x, y \in \Lambda_{n}} \iint_{I_{\beta} \times I_{\beta}} d s d t G_{\beta}((x, s),(y, t)) \rightarrow 0 \text { as } n \rightarrow \infty .
$$


Similarly, for $\beta=\infty$ we have that

$$
\begin{aligned}
G_{N, r}((x, s),(y, t)) & \rightarrow \frac{1}{(2 \pi)^{d+1}} \int_{(-\pi, \pi]^{d}} d p \int_{\mathbb{R}} d q \frac{e^{-i p \cdot(x-y)} e^{-i q(s-t)}}{E_{\lambda, \delta}(p, q)} \\
& =: G_{\infty}((x, s),(y, t)), \text { as } N, r \rightarrow \infty,
\end{aligned}
$$

and hence using (30) that

(33) $\frac{1}{(2 n)^{2 d} r^{2}} \sum_{x, y \in \Lambda_{n}} \iint_{I_{r} \times I_{r}} d s d t G_{\infty}((x, s),(y, t)) \rightarrow 0$ as $n, r \rightarrow \infty$.

We now show how (32) and (33) imply (27) and (28), respectively. Note that by Fourier inversion

$$
c_{N, r}(x, t)=\frac{1}{(2 N)^{d} r} \sum_{k \in \Lambda_{N}^{\star}} \sum_{\ell \in I_{r}^{\star}} \hat{c}_{N, r}(k, \ell) e^{-i k \cdot x} e^{-i \ell t} .
$$

Let $v: K(N, r) \rightarrow \mathbb{C}$ be an aribtrary bounded, measurable function. It follows that

$$
\begin{gathered}
\sum_{x, y \in \Lambda_{N}} \iint_{I_{r} \times I_{r}} d s d t v(x, s) \overline{v(y, t)} c_{N, r}(x-y, s-t) \\
=\frac{1}{(2 N)^{d} r} \sum_{\xi \in K_{N, r}^{\star}} \hat{c}_{N, r}(\xi)\left|z_{v}(\xi)\right|^{2},
\end{gathered}
$$

where

$$
z_{v}(k, \ell)=\sum_{x \in \Lambda_{N}} \int_{I_{r}} v(x, s) e^{-i k \cdot x} e^{-i \ell s} d s .
$$

Using the infrared bound (26),

$$
\sum_{\xi \in K_{N, r}^{\star}} \hat{c}_{N, r}(\xi)\left|z_{v}(\xi)\right|^{2} \leq \sum_{\xi \in K_{N, r}^{\star} \backslash\{0\}} \frac{1}{E_{\lambda, \delta}(\xi)}\left|z_{v}(\xi)\right|^{2}+\hat{c}_{N, r}(0)\left|z_{v}(0)\right|^{2} .
$$

Note that

$$
\hat{c}_{N, r}(0)=\sum_{x \in \Lambda_{N}} \int_{I_{r}} c_{N, r}(x, t) d t=: \chi_{N, r}^{\mathfrak{p}, \mathfrak{p}}
$$

equals the (finite-volume) susceptibility. Interchanging the order of summation again thus gives

$$
\begin{aligned}
& \frac{1}{(2 N)^{d} r} \sum_{\xi \in K_{N, r}^{\star}} \hat{c}_{N, r}(\xi)\left|z_{v}(\xi)\right|^{2} \\
& \leq \sum_{x, y \in \Lambda_{N}} \iint_{I_{r} \times I_{r}} v(x, s) \overline{v(y, t)} G_{N, r}((x, s),(y, t))+\frac{\left|z_{v}(0)\right|^{2}}{(2 N)^{d} r} \chi_{N, r}^{\mathfrak{p}, \mathfrak{p}} .
\end{aligned}
$$

Let $N_{0}<N$, and as usual let $r_{0}<r$ if $\beta=\infty$, alternatively $r_{0}=$ $r=\beta$ if $\beta<\infty$. Set

$$
v(x, s)=\mathbb{I}\left\{x \in \Lambda_{N_{0}}, s \in I_{r_{0}}\right\} .
$$


In what follows we use the same notation $\langle\cdot\rangle_{N, r}^{\mathfrak{f}}$ for both $\langle\cdot\rangle_{N, r}^{\mathfrak{f}, f}$ (in the case $\beta=\infty$ ) and $\langle\cdot\rangle_{N, \beta}^{\mathfrak{f}, \mathfrak{p}}$ (in the case $\beta<\infty$ ). We also write $\langle\cdot\rangle_{\lambda, \beta}^{(\mathfrak{f})}$ for both infinite-volume limits $\langle\cdot\rangle_{\lambda, \infty}^{(\mathfrak{f}, \mathfrak{f})}$ and $\langle\cdot\rangle_{\lambda, \beta}^{(\mathfrak{f}, \mathfrak{p})}$. By the monotonicity (8) of correlation functions we have that

$$
c_{N, r}(x-y, s-t)=\langle\sigma(x, s) \sigma(y, t)\rangle_{N, r}^{\mathfrak{p}, \mathfrak{p}} \geq\langle\sigma(x, s) \sigma(y, t)\rangle_{N, r}^{\mathfrak{f}} .
$$

Thus for our choice of $v$ we have that the left-hand-side of (34) is at least

$$
\sum_{x, y \in \Lambda_{N_{0}}} \iint_{I_{r_{0}} \times I_{r_{0}}}\langle\sigma(x, s) \sigma(y, t)\rangle_{N, r}^{\uparrow} d s d t .
$$

By (35) it follows that

$$
\begin{aligned}
& \sum_{x, y \in \Lambda_{N_{0}}} \iint_{I_{r_{0}} \times I_{r_{0}}}\langle\sigma(x, s) \sigma(y, t)\rangle_{N, r}^{\mathfrak{f}} d s d t \\
& \quad \leq \sum_{x, y \in \Lambda_{N_{0}}} \iint_{I_{r_{0}} \times I_{r_{0}}} d s d t G_{N, r}((x, s),(y, t))+\frac{\left(2 N_{0}\right)^{d} r_{0}}{(2 N)^{d} r} \chi_{N, r}^{\mathfrak{p},} .
\end{aligned}
$$

Now let $\lambda<\lambda_{\mathrm{c}}$. This implies that $\langle\cdot\rangle_{\lambda, \beta}^{(\mathfrak{f})}$ is the unique infinite-volume limit of the measures $\langle\cdot\rangle_{N, r}^{\mathfrak{s}, \mathrm{t}}$, and by finiteness of the susceptibility [9, Theorem 6.6] and the dominated convergence theorem we have that

$$
\chi_{N, r}^{\mathfrak{p}, \mathfrak{p}} \rightarrow \sum_{x \in \mathbb{Z}^{d}} \int_{I_{\beta}}\langle\sigma(0,0) \sigma(x, t)\rangle_{\lambda, \beta}^{(\mathfrak{f})} d t<\infty,
$$

as $N \rightarrow \infty$ (for $\beta=r<\infty)$ or $N, r=2 N \rightarrow \infty$ (for $\beta=\infty)$. Hence, letting $N \rightarrow \infty$ or $N, r \rightarrow \infty$ as appropriate, we obtain from (36) that for all $\lambda<\lambda_{\mathrm{c}}$ we have

$$
\begin{aligned}
\sum_{x, y \in \Lambda_{N_{0}}} & \iint_{I_{r_{0}} \times I_{r_{0}}}\langle\sigma(x, s) \sigma(y, t)\rangle_{\lambda, \beta}^{(\mathfrak{f})} d s d t \\
& \leq \sum_{x, y \in \Lambda_{N_{0}}} \iint_{I_{r_{0}} \times I_{r_{0}}} G_{\beta}((x, s),(y, t)) d s d t
\end{aligned}
$$

Letting $\lambda \uparrow \lambda_{\mathrm{c}}$ and using the fact that $\langle\sigma(x, s) \sigma(y, t)\rangle_{\lambda, \beta}^{(\mathfrak{f})}$ is left-continuous in $\lambda$ (since any two increasing limits can be interchanged), we get that (37) holds also with $\lambda=\lambda_{\mathrm{c}}$. Letting $N_{0} \rightarrow \infty$ or $N_{0}, r_{0} \rightarrow \infty$ as appropriate we get from (32) and (33) that

$$
\frac{1}{\left(2 N_{0}\right)^{2 d} r_{0}^{2}} \sum_{x, y \in \Lambda_{N_{0}}} \iint_{I_{r_{0}} \times I_{r_{0}}}\langle\sigma(x, s) \sigma(y, t)\rangle_{\lambda_{\mathrm{c}}, \beta}^{(\mathfrak{f})} d s d t \rightarrow 0 .
$$

Using translation-invariance and nonnegativity of $\langle\sigma(x, s) \sigma(y, t)\rangle_{\lambda_{\mathrm{c}}, \beta}^{(\mathfrak{f})}$, the results (27) and (28) follow. 
4.2. Proof of Theorem 1.1. From this point the argument is almost identical to that for the classical model [2], however it is also short and elegant so we include the remaining steps. We begin by deducing from Lemma 4.1 the following consequence for the number $U$ of unbounded components under the measure $\overline{\mathbb{P}}$.

Proposition 4.2. Under the conditions in Lemma 4.1 and for $\lambda=$ $\lambda_{\mathrm{c}}$ we have that $\overline{\mathbb{P}}(U=0)=1$.

Proof. Recall our convention on boundary conditions for the measure $\overline{\mathbb{P}}_{N, r}$ : if $\beta<\infty$ we write $\mathfrak{t}_{1}=\mathfrak{t}_{2}=\mathfrak{p}$, if $\beta=\infty$ we write $\mathfrak{t}_{1}=\mathfrak{f}$ and $\mathfrak{t}_{2}=\mathfrak{w}$. By the definition of $\overline{\mathbb{P}}_{N, r}$ and the Switching Lemma 2.1 we have for any $N, r$ and $(x, s),(y, t) \in K(N, r)$ that

$$
\begin{aligned}
\overline{\mathbb{P}}_{N, r}((x, s) \leftrightarrow(y, t)) & =\langle\sigma(x, s) \sigma(y, t)\rangle_{N, r}^{\mathfrak{f}, \mathfrak{t}_{1}}\langle\sigma(x, s) \sigma(y, t)\rangle_{N, r}^{\mathfrak{w}, t_{2}} \\
& \leq\langle\sigma(x, s) \sigma(y, t)\rangle_{N, r}^{\mathfrak{f}, \mathfrak{t}_{1}} .
\end{aligned}
$$

By the convergence of the correlation function and using Proposition 3.1 (and a small, but standard, additional argument) we get that

$$
\overline{\mathbb{P}}((x, s) \leftrightarrow(y, t)) \leq\langle\sigma(x, s) \sigma(y, t)\rangle_{\lambda_{c}, \beta}^{\left(\mathfrak{f}, \mathfrak{t}_{1}\right)}
$$

Writing $\{(x, s) \leftrightarrow \infty\}$ for the event that $(x, s)$ lies in an unbounded component we have using Jensen's inequality and the fact that $U \leq 1$ (Proposition 3.3) that

$$
\begin{aligned}
\left(r\left|\Lambda_{N}\right| \overline{\mathbb{P}}((0,0) \leftrightarrow \infty)\right)^{2} & \leq \overline{\mathbb{P}}\left(\left(\sum_{x \in \Lambda_{N}} \int_{I_{r}} \mathbb{I}\{(x, s) \leftrightarrow \infty\} d s\right)^{2}\right) \\
& =\sum_{x, y \in \Lambda_{N}} \iint_{I_{r} \times I_{r}} \overline{\mathbb{P}}((x, s),(y, t) \leftrightarrow \infty) d s d t \\
& \leq \sum_{x, y \in \Lambda_{N}} \iint_{I_{r} \times I_{r}} \overline{\mathbb{P}}((x, s) \leftrightarrow(y, t)) d s d t \\
& =r\left|\Lambda_{N}\right| \sum_{x \in \Lambda_{N}} \int_{I_{r}} \overline{\mathbb{P}}((0,0) \leftrightarrow(x, s)) d s .
\end{aligned}
$$

Using (38) we deduce that

$$
\overline{\mathbb{P}}((0,0) \leftrightarrow \infty)^{2} \leq \frac{1}{r\left|\Lambda_{N}\right|} \sum_{x \in \Lambda_{N}} \int_{I_{r}}\langle\sigma(0,0) \sigma(x, s)\rangle_{\lambda_{\mathrm{c}}, \beta}^{\left(\mathfrak{f}, \mathfrak{t}_{1}\right)} d s .
$$

Letting $N \rightarrow \infty$ or $N, r \rightarrow \infty$ as appropriate, and using Lemma 4.1, the result follows.

Turning to the final steps in the proof of Theorem 1.1, we recall from Proposition 2.2 that for each $(x, t) \in \mathbb{K}_{\beta}$ there is a constant $C_{(x, t)}$ such that for all $N, r$ we have

$$
\langle\sigma(0,0) \sigma(x, t)\rangle_{N, r}^{\mathfrak{w}, \mathfrak{t}_{2}}-\langle\sigma(0,0) \sigma(x, t)\rangle_{N, r}^{\mathfrak{f}, \mathfrak{t}_{1}} \leq C_{(x, t)} \overline{\mathbb{P}}_{N, r}((0,0) \leftrightarrow \Gamma) .
$$


Also note that for all $N_{0} \leq N$ and $r_{0} \leq r$ we have that $\overline{\mathbb{P}}_{N, r}((0,0) \leftrightarrow$ $\Gamma) \leq \overline{\mathbb{P}}_{N, r}\left((0,0) \leftrightarrow \partial K\left(N_{0}, r_{0}\right)\right)$, since any path to $\Gamma$ must leave $K\left(N_{0}, r_{0}\right)$. Letting $N \rightarrow \infty$ (respectively, $N, r \rightarrow \infty$ ) and then $N_{0} \rightarrow \infty$ (respectively, $\left.N_{0}, r_{0} \rightarrow \infty\right)$ it follows from Proposition 4.2 that

$$
\langle\sigma(0,0) \sigma(x, t)\rangle_{\lambda_{c}, \beta}^{\left(\mathfrak{w}, \mathfrak{t}_{2}\right)}-\langle\sigma(0,0) \sigma(x, t)\rangle_{\lambda_{c}, \beta}^{\left(\mathfrak{f}, \mathfrak{t}_{1}\right)} \leq C_{(x, t)} \overline{\mathbb{P}}((0,0) \leftrightarrow \infty)=0 .
$$

Thus $\langle\sigma(0,0) \sigma(x, t)\rangle_{\lambda_{\mathrm{c}}, \beta}^{\left(\mathfrak{w}, \mathfrak{t}_{2}\right)}=\langle\sigma(0,0) \sigma(x, t)\rangle_{\lambda_{\mathrm{c}}, \beta}^{\left(\mathfrak{f}, \mathfrak{t}_{1}\right)}$. By translation-invariance and the Griffiths inequality (proved in detail for the present model in [7, Lemma 2.2.20]) it follows that

$$
\begin{aligned}
\left(\langle\sigma(0,0)\rangle_{\lambda_{c}, \beta}^{\left(\mathfrak{w}, \mathfrak{t}_{2}\right)}\right)^{2} & =\langle\sigma(0,0)\rangle_{\lambda_{c}, \beta}^{\left(\mathfrak{w}, \mathfrak{t}_{2}\right)}\langle\sigma(x, t)\rangle_{\lambda_{c}, \beta}^{\left(\mathfrak{w}, \mathfrak{t}_{2}\right)} \leq\langle\sigma(0,0) \sigma(x, t)\rangle_{\lambda_{c}, \beta}^{\left(\mathfrak{w}, \mathfrak{t}_{2}\right)} \\
& =\langle\sigma(0,0) \sigma(x, t)\rangle_{\lambda_{c}, \beta}^{\left(\mathfrak{f}, \mathfrak{t}_{1}\right)}
\end{aligned}
$$

Thus using Lemma 4.1 again, if $\beta<\infty$ and $d \geq 3$ or $\beta=\infty$ and $d \geq 2$ then

$$
\left(\langle\sigma(0,0)\rangle_{\lambda_{c}, \beta}^{\left(\mathfrak{w}, \mathfrak{t}_{2}\right)}\right)^{2} \leq \frac{1}{r\left|\Lambda_{N}\right|} \sum_{x \in \Lambda_{N}} \int_{I_{r}}\langle\sigma(0,0) \sigma(x, t)\rangle_{\lambda_{c}, \beta}^{\left(\mathfrak{f}, \mathfrak{t}_{2}\right)} d t \rightarrow 0
$$

hence by (91) we have $M_{\beta}^{+}\left(\lambda_{\mathrm{c}}\right)=\langle\sigma(0,0)\rangle_{\lambda_{\mathrm{c}}, \beta}^{\left(\mathfrak{w}, \mathfrak{t}_{2}\right)}=0$ as claimed.

\section{AppendiX: MIXING IN THE SPACE-TIME SPIN REPRESENTATION}

In this section we prove mixing results for the infinite-volume spacetime spin measures $\mu_{\beta}^{(\mathfrak{s}, \mathfrak{t})}$ defined in Section 2.1. As usual we let $\mathfrak{t}=\mathfrak{p}$ if $\beta<\infty$ and $\mathfrak{t} \in\{\mathfrak{f}, \mathfrak{w}\}$ if $\beta=\infty$. As a shorthand we write

$$
\mu^{(\mathfrak{w})}=\left\{\begin{array}{ll}
\mu_{\infty}^{(\mathfrak{w}, \mathfrak{w})} & \text { if } \beta=\infty, \\
\mu_{\beta}^{(\mathfrak{w}, \mathfrak{p})} & \text { if } \beta<\infty,
\end{array} \quad \mu^{(\mathfrak{f})}= \begin{cases}\mu_{\infty}^{(\mathfrak{f}, \mathfrak{f})} & \text { if } \beta=\infty, \\
\mu_{\beta}^{(\mathfrak{f}, \mathfrak{p})} & \text { if } \beta<\infty,\end{cases}\right.
$$

and $\langle\cdot\rangle^{(\mathfrak{w})},\langle\cdot\rangle^{(\mathfrak{f})}$ for the corresponding expectation operators. For simplicity of presentation we focus on the case $\beta=\infty$, similar results and constructions hold for the case $\beta<\infty$.

To state and prove our mixing results we need to be precise about the topological set-up. We define a metric $d$ on $\Sigma$ as follows. Firstly, for each $n \geq 1$ define a 'local' metric

$$
d_{n}\left(\sigma, \sigma^{\prime}\right)=\sum_{x \in \Lambda_{n}} \int_{I_{n}}\left|\sigma(x, t)-\sigma^{\prime}(x, t)\right| d t, \quad \sigma, \sigma^{\prime} \in \Sigma,
$$

and then extend this in a standard way by letting

$$
d\left(\sigma, \sigma^{\prime}\right)=\sum_{n \geq 0} 2^{-n} \frac{d_{n}\left(\sigma, \sigma^{\prime}\right)}{1+d_{n}\left(\sigma, \sigma^{\prime}\right)} .
$$

Recall that a function $F: \Sigma \rightarrow \mathbb{R}$ is

- uniformly continuous if for each $\varepsilon>0$ there is $\delta>0$ such that if $d\left(\sigma, \sigma^{\prime}\right)<\delta$ then $\left|F(\sigma)-F\left(\sigma^{\prime}\right)\right|<\varepsilon$

- even if $F(\sigma)=F(-\sigma)$ for all $\sigma \in \Sigma$. 
We will prove the following:

Lemma 5.1. Let $0<\beta \leq \infty$ and let $C_{1}, C_{2}: \Sigma \rightarrow \mathbb{R}$ be bounded, uniformly continuous functions. Then

$$
\left.\lim _{\|x\| \rightarrow \infty}\left\langle C_{1}(\sigma)\left[C_{2} \circ \tau_{x}\right](\sigma)\right]\right\rangle^{(\mathfrak{w})}=\left\langle C_{1}(\sigma)\right\rangle^{(\mathfrak{w})}\left\langle C_{2}(\sigma)\right\rangle^{(\mathfrak{w})} .
$$

If, in addition, $C_{1}, C_{2}$ are even then also

$$
\left.\lim _{\|x\| \rightarrow \infty}\left\langle C_{1}(\sigma)\left[C_{2} \circ \tau_{x}\right](\sigma)\right]\right\rangle^{(\mathfrak{f})}=\left\langle C_{1}(\sigma)\right\rangle^{(\mathfrak{f})}\left\langle C_{2}(\sigma)\right\rangle^{(\mathfrak{f})} .
$$

The proof follows the strategy in the appendix of [2], and is based on first proving the statement for functions of the form $C(\sigma)=\sigma_{A}$ using the Griffiths inequality and then extending to more general functions using the Stone-Weierstrass theorem. However, there are two diffculties associated with this approach: firstly, the function $C(\sigma)=\sigma_{A}$ is not continuous; secondly, $\Sigma$ is not compact. (The locally compact version of the Stone-Weierstrass theorem is not appropriate since the functions $C$ we want to consider do not 'vanish at infinity'.) Nonetheless, we have the following result. Let $\mathcal{G}$ denote the (real) algebra of functions generated by the monomials of the form $\sigma_{A}$ for finite $A \subseteq \mathbb{K}$. For tidier notation we drop the superscript ${ }^{(\mathfrak{w})}$ or ${ }^{(\mathfrak{f})}$ in the following result, which holds for both cases.

Proposition 5.2. Suppose $F: \Sigma \rightarrow \mathbb{R}$ is a bounded and measurable function such that for all $G \in \mathcal{G}$

$$
\lim _{\|x\| \rightarrow \infty}\left\langle G(\sigma)\left[F \circ \tau_{x}\right](\sigma)\right\rangle=\langle G(\sigma)\rangle\langle F(\sigma)\rangle .
$$

Then for all bounded, uniformly continuous $C: \Sigma \rightarrow \mathbb{R}$ we also have

$$
\lim _{\|x\| \rightarrow \infty}\left\langle C(\sigma)\left[F \circ \tau_{x}\right](\sigma)\right\rangle=\langle C(\sigma)\rangle\langle F(\sigma)\rangle .
$$

Proof. For each $\delta>0$ let $\Sigma_{\delta}$ denote the set of functions $\sigma \in \Sigma$ which are constant on each interval of the form $\{x\} \times[k \delta,(k+1) \delta)$ for $k \in \mathbb{Z}$. Then (by a diagonal argument or otherwise) $\Sigma_{\delta}$ is compact. Define a mapping $\Sigma \rightarrow \Sigma_{\delta}$ by letting $\sigma \mapsto \sigma_{\delta}$ where $\sigma_{\delta}(x, t)=\sigma(x, \delta\lfloor t / \delta\rfloor)$, and for $F: \Sigma \rightarrow \mathbb{R}$ let $F_{\delta}: \Sigma \rightarrow \mathbb{R}$ be given by $F_{\delta}(\sigma)=F\left(\sigma_{\delta}\right)$. Note that if $G \in \mathcal{G}$ then $G_{\delta} \in \mathcal{G}$. Let $\mathcal{C}_{\delta}$ denote the set of continuous functions $\Sigma_{\delta} \rightarrow \mathbb{R}$ and $\mathcal{G}_{\delta}$ the set of restrictions of functions in $\mathcal{G}$ to $\Sigma_{\delta}$. Then $\mathcal{G}_{\delta}$ is an algebra of functions in $\mathcal{C}_{\delta}$, and $\mathcal{G}_{\delta}$ separates the points of $\Sigma_{\delta}$ (if $\sigma, \sigma^{\prime} \in \Sigma_{\delta}$ differ at the point $(x, k \delta)$ then, by definition, $\left.\sigma(x, k \delta) \neq \sigma^{\prime}(x, k \delta)\right)$. Thus by the Stone-Weierstrass theorem $\mathcal{C}_{\delta}$ is the uniform closure of $\mathcal{G}_{\delta}$, meaning that for each bounded, uniformly continuous $C: \Sigma \rightarrow \mathbb{R}$ and each $\varepsilon>0$ there is $G \in \mathcal{G}$ such that

$$
\sup _{\sigma \in \Sigma}\left|G_{\delta}(\sigma)-C_{\delta}(\sigma)\right|=\sup _{\sigma \in \Sigma_{\delta}}\left|G_{\delta}(\sigma)-C_{\delta}(\sigma)\right|<\varepsilon .
$$


Let $M$ be a uniform upper bound on both $|F|$ and $|C|$. We have that

$$
\begin{aligned}
\mid\left\langle C(\sigma)\left[F \circ \tau_{x}\right](\sigma)\right\rangle & -\left\langle G_{\delta}(\sigma)\left[F \circ \tau_{x}\right](\sigma)\right\rangle \mid \\
& \leq M\left\langle\left|G_{\delta}(\sigma)-C_{\delta}(\sigma)\right|\right\rangle+M\left\langle\left|C(\sigma)-C_{\delta}(\sigma)\right|\right\rangle \\
& \leq M \varepsilon+M\left\langle\left|C(\sigma)-C_{\delta}(\sigma)\right|\right\rangle .
\end{aligned}
$$

For $\eta>0$ sufficiently small,

$$
\begin{aligned}
\left|C(\sigma)-C_{\delta}(\sigma)\right|= & \left|C(\sigma)-C\left(\sigma_{\delta}\right)\right| \mathbb{I}\left\{d\left(\sigma, \sigma_{\delta}\right)<\eta\right\} \\
& +\left|C(\sigma)-C_{\delta}(\sigma)\right| \mathbb{I}\left\{d\left(\sigma, \sigma_{\delta}\right) \geq \eta\right\} \\
\leq & \varepsilon+2 M \mathbb{I}\left\{d\left(\sigma, \sigma_{\delta}\right) \geq \eta\right\} .
\end{aligned}
$$

Thus $\left\langle\left|C(\sigma)-C_{\delta}(\sigma)\right|\right\rangle \leq \varepsilon+2 M \mu\left(d\left(\sigma, \sigma_{\delta}\right) \geq \eta\right)$, and the last probability converges to 0 as $\delta \downarrow 0$ (for example along a sequence of the form $\left.\delta=2^{-m}\right)$. Hence (39) can be made arbitrarily small, uniformly in $x$. The same bound applies to $\left|\langle C(\sigma)\rangle\langle F(\sigma)\rangle-\left\langle G_{\delta}(\sigma)\right\rangle\langle F(\sigma)\rangle\right|$. Since $G_{\delta} \in \mathcal{G}$, the result follows.

Remark 5.3. Proposition 5.2 holds also if we assume in addition that $F, G$ and $C$ are even functions. To prove this in detail one may pass to the quotient space $\Sigma / \sim$, where the equivalence relation $\sim$ consists of all pairs $\{\sigma,-\sigma\}$ for $\sigma \in \Sigma$. An even function on $\Sigma$ may be identified with a function on $\Sigma / \sim$ and this identifies continuous functions with continuous functions. (This uses the fact that the mapping $\sigma \mapsto-\sigma$ is an isometry and [10, Lemma 3.3.6].) The subspace $\Sigma_{\delta} / \sim$ is compact and the even functions in $\mathcal{G}$ separate the points of $\Sigma_{\delta} / \sim$, so we may apply the Stone-Weierstrass theorem in the same way as in Proposition 5.2. The remaining estimates are the same.

Proof of Lemma 5.1. We allow ourselves to be rather brief and omit some details. For the boundary condition $\mathfrak{w}$ it suffices to show that for all finite sets $A, B \subseteq \mathbb{K}$,

$$
\left\langle\sigma_{A} \sigma_{B+x}\right\rangle^{(\mathfrak{w})} \rightarrow\left\langle\sigma_{A}\right\rangle^{(\mathfrak{w})}\left\langle\sigma_{B}\right\rangle^{(\mathfrak{w})} \text { as }\|x\| \rightarrow \infty,
$$

by Proposition 5.2 and linearity. For the boundary condition $\mathfrak{f}$ we need to show that (40) holds (with $\mathfrak{w}$ replaced by $\mathfrak{f}$ ) when $A$ and $B$ are sets of even size, by Remark 5.3.

Fix $N_{0}<N$ and $r_{0}<r$ large enough that $A, B \subseteq K\left(N_{0}, r_{0}\right)$, and write $K$ for $K(N, r)$ and $K_{0}$ for $K\left(N_{0}, r_{0}\right)$. We begin by showing that for each bounded, measurable function $h: K_{0} \rightarrow[0, \infty)$ we have

$$
\begin{aligned}
\lim _{\|x\| \rightarrow \infty}\left\langle\sigma_{A} \exp \right. & \left.\left(\sum_{y \in \Lambda_{N_{0}}} \int_{I_{r_{0}}} h(y, t) \sigma(y+x, t) d t\right)\right\rangle^{(\mathfrak{w})} \\
& =\left\langle\sigma_{A}\right\rangle^{(\mathfrak{w})}\left\langle\exp \left(\sum_{y \in \Lambda_{N_{0}}} \int_{I_{r_{0}}} h(y, t) \sigma(y, t) d t\right)\right\rangle^{(\mathfrak{w})} .
\end{aligned}
$$


To go from (41) to (40) one expands the exponentials as a power series. Using the fact that (41) holds for arbitrary $h$ and that correlation functions of the form $\left\langle\sigma\left(y_{1}, t_{1}\right) \cdots \sigma\left(y_{k}, t_{k}\right)\right\rangle^{(\mathfrak{w})}$ are continuous in $t_{1}, \ldots, t_{k}$ one may deduce pointwise convergence of the form (40) from the corresponding convergence of repeated sums and integrals over $y_{1}, \ldots, y_{k}$ and $t_{1}, \ldots, t_{k}$.

We now show (41). Write $K(x)=\Lambda_{\|x\|-N_{0}} \times I_{r_{0}+\|x\|}$ where $x \in \mathbb{Z}^{d}$ is fixed with $\|x\|$ large enough that $A \subseteq K(x)$. Let $N, r$ be large enough that $K_{0}+x \subseteq K$. Write

$$
h(\sigma)=\sum_{y \in \Lambda_{N_{0}}} \int_{I_{r_{0}}} h(y, t) \sigma(y, t) d t
$$

and let $\langle\cdot\rangle_{K ; h \circ \tau_{x}}^{\mathfrak{w}}$ denote the wired space-time Ising measure defined as in (5) - (66) but with the additional term

$$
h\left(\tau_{x}(\sigma)\right)=\sum_{y \in \Lambda_{N_{0}}} \int_{I_{r_{0}}} h(y, t) \sigma(y+x, t) d t
$$

in the exponential. Using the shorthand $\langle\cdot\rangle_{K}^{\mathfrak{w}}$ for $\langle\cdot\rangle_{N, r}^{\mathfrak{w}, \mathfrak{w}}$ we have

$$
\left\langle\sigma_{A} \exp \left(h\left(\tau_{x}(\sigma)\right)\right)\right\rangle_{K}^{\mathfrak{w}}=\left\langle\sigma_{A}\right\rangle_{K ; h \circ \tau_{x}}^{\mathfrak{w}}\left\langle\exp \left(h\left(\tau_{x}(\sigma)\right)\right)\right\rangle_{K}^{\mathfrak{w}} .
$$

The Griffiths inequality (see [7, Lemma 2.2.20] for a proof for the present model) implies that the correlation $\left\langle\sigma_{A}\right\rangle_{K ; h \circ \tau_{x}}^{\mathfrak{w}}$ is increasing when viewed as a function of $h$ (under pointwise ordering of $h$ ). Comparison with the case $h \equiv 0$ gives

$$
\left\langle\sigma_{A}\right\rangle_{K ; h \circ \tau_{x}}^{\mathfrak{w}} \geq\left\langle\sigma_{A}\right\rangle_{K}^{\mathfrak{w}} \text {. }
$$

If we let $h(y, t) \rightarrow \infty$ for all $(y, t) \in K_{0}$ then $\langle\cdot\rangle_{K ; h \circ \tau_{x}}^{\mathfrak{w}}$ converges to a state corresponding to 'wiring' the region $K_{0}+x$, and we deduce that

$$
\left\langle\sigma_{A}\right\rangle_{K ; h \circ \tau_{x}}^{\mathfrak{w}} \leq\left\langle\sigma_{A}\right\rangle_{K(x)}^{\mathfrak{w}}
$$

(cf. [7, Lemma 2.2.22]). Letting $N, r \rightarrow \infty$ and applying translationinvariance we obtain

$$
\begin{aligned}
\left\langle\sigma_{A}\right\rangle^{(\mathfrak{w})}\langle\exp (h(\sigma))\rangle^{(\mathfrak{w})} & \leq\left\langle\sigma_{A} \exp \left(h\left(\tau_{x}(\sigma)\right)\right)\right\rangle^{(\mathfrak{w})} \\
& \leq\left\langle\sigma_{A}\right\rangle_{K(x)}^{\mathfrak{w}}\langle\exp (h(\sigma))\rangle^{(\mathfrak{w})}
\end{aligned}
$$

Letting $\|x\| \rightarrow \infty$ we have $K(x) \uparrow \mathbb{K}$ and hence (41) follows.

For the case of boundary condition $\mathfrak{f}$ let $J: F\left(N_{0}, r_{0}\right) \rightarrow[-\lambda, 0]$ be measurable and $q: K_{0} \rightarrow[0, \infty)$ be bounded and measurable. Write

$$
J(\sigma)=\sum_{y z \in \mathcal{E}_{N_{0}}} \int_{I_{r_{0}}} J(y z, t) \sigma(y, t) \sigma(z, t) d t,
$$

and (recalling the process $D$ of discontinuities of $\sigma$ )

$$
q(\sigma)=\sum_{(y, t) \in D \cap K_{0}} q(y, t) .
$$


Note that $q(\sigma)$ is a function of $D$ only, and we may therefore write $q(D)$ in place of $q(\sigma)$. With this notation we have $q\left(\tau_{x}(\sigma)\right)=q\left(\tau_{-x}(D)\right)$. Let $\langle\cdot\rangle_{K ;(J, q) \circ \tau_{x}}^{\mathfrak{f}}$ denote the measure defined as in (5) -(6) but with the additional term $J\left(\tau_{x}(\sigma)\right)+q\left(\tau_{x}(\sigma)\right)$ in the exponential. We have that

$$
\begin{aligned}
\left\langle\sigma _ { A } \operatorname { e x p } \left( J\left(\tau_{x}(\sigma)\right)\right.\right. & \left.\left.+q\left(\tau_{x}(\sigma)\right)\right)\right\rangle_{K}^{\mathfrak{f}} \\
& =\left\langle\sigma_{A}\right\rangle_{K ;(J, q) \circ \tau_{x}}^{\mathfrak{f}}\left\langle\exp \left(J\left(\tau_{x}(\sigma)\right)+q\left(\tau_{x}(\sigma)\right)\right)\right\rangle_{K}^{\mathfrak{f}} .
\end{aligned}
$$

By standard properties of Poisson processes, $\langle\cdot\rangle_{K ;(J, q) \circ \tau_{x}}^{\mathfrak{f}}$ may alternatively be obtained by first modifying the intensity of $D$ under the apriori measure $E_{N, r}$ from the constant intensity $\delta$ to the variable intensity

$$
\delta(y, t)=\delta \exp \left(q(y-x, t) \mathbb{I}\left\{(y, t) \in K_{0}+x\right\}\right),
$$

and then having only the additional term $J\left(\tau_{x}(\sigma)\right)$ in the exponential. Thus the correlation $\left\langle\sigma_{A}\right\rangle_{K ;(J, q) \circ \tau_{x}}^{\mathfrak{f}}$ is increasing in $J$ and decreasing in $q$, and comparison with the cases $J \equiv-\lambda, q \equiv \infty$, respectively $J \equiv 0$, $q \equiv 0$, gives

$$
\left\langle\sigma_{A}\right\rangle_{K(x)}^{\mathfrak{f}} \leq\left\langle\sigma_{A}\right\rangle_{K ;(J, q) \circ \tau_{x}}^{\mathfrak{f}} \leq\left\langle\sigma_{A}\right\rangle_{K}^{\mathfrak{f}} .
$$

Similarly to (41) we deduce that

$\lim _{\|x\| \rightarrow \infty}\left\langle\sigma_{A} \exp \left(J\left(\tau_{x}(\sigma)\right)+q\left(\tau_{x}(\sigma)\right)\right)\right\rangle^{(\mathfrak{f})}=\left\langle\sigma_{A}\right\rangle^{(\mathfrak{f})}\langle\exp (J(\sigma)+q(\sigma))\rangle^{(\mathfrak{f})}$.

Expanding the exponential $\exp (J(\sigma))$ as for (40) we deduce that for all finite $B \subseteq \mathbb{F}$,

$$
\left\langle\sigma_{A} \sigma_{B+x} \exp \left(q\left(\tau_{x}(\sigma)\right)\right)\right\rangle^{(\mathfrak{f})} \rightarrow\left\langle\sigma_{A}\right\rangle^{(\mathfrak{f})}\left\langle\sigma_{B} \exp (q(\sigma))\right\rangle^{(\mathfrak{f})} \text { as }\|x\| \rightarrow \infty .
$$

Let $x_{1}, \ldots, x_{n} \in \mathbb{Z}^{d}$ and let $s_{1}<t_{1}, \ldots, s_{n}<t_{n}$ be real numbers such that all points of the form $\left(x_{j}, s_{j}\right)$ or $\left(x_{j}, t_{j}\right)$ are distinct, and let $B^{\prime}$ be the set of these points. (Thus $B^{\prime} \cap(\{x\} \times \mathbb{R})$ has even size for all $x \in \mathbb{Z}^{d}$.) One may deduce from (46) that for any such set $B^{\prime} \subseteq \mathbb{K}$ we have that

$$
\left\langle\sigma_{A} \sigma_{B+x} \sigma_{B^{\prime}+x}\right\rangle^{(\mathfrak{f})} \rightarrow\left\langle\sigma_{A}\right\rangle^{(\mathfrak{f})}\left\langle\sigma_{B} \sigma_{B^{\prime}}\right\rangle^{(\mathfrak{f})} \text { as }\|x\| \rightarrow \infty .
$$

This proves the claim of the lemma for the boundary condition $\mathfrak{f}$ since for any set $B \subseteq \mathbb{K}$ of even size one may write $\sigma_{B}=\sigma_{B^{\prime}} \sigma_{B^{\prime \prime}}$ for some set $B^{\prime}$ as above, and some finite $B^{\prime \prime} \subseteq \mathbb{F}$.

One way to see that (46) implies (47) is as follows (we give only a sketch). One may see (46) as a result about convergence of the Laplace functionals of the point processes $\tau_{x}^{-1}(D) \cap K_{0}$ with certain 'skewed' distributions. Using Theorem 11.1.VI and Proposition 11.1.VII of [14] it follows that

$$
\left\langle\sigma_{A} \sigma_{B+x} \mathbb{I}\left\{\tau_{x}^{-1}(D) \in \mathcal{C}\right\}\right\rangle^{(\mathfrak{f})} \rightarrow\left\langle\sigma_{A}\right\rangle^{(\mathfrak{f})}\left\langle\sigma_{B} \mathbb{I}\{D \in \mathcal{C}\}\right\rangle^{(\mathfrak{f})}
$$


for each 'stochastic continuity set' $\mathcal{C}$. These sets include the events

$$
\mathcal{C}_{j}=\left\{\left|D \cap\left(\left\{x_{j}\right\} \times\left[s_{j}, t_{j}\right)\right)\right| \text { is even }\right\},
$$

and using the identity $\sigma\left(x_{j}, s_{j}\right) \sigma\left(x_{j}, t_{j}\right)=2 \mathbb{I}_{\mathcal{C}_{j}}-1$ one may write $\sigma_{B^{\prime}}$ as a linear combination of terms of the form $\mathbb{1}\left\{\bigcap_{j \in J} \mathcal{C}_{j}\right\}$ for $J \subseteq$ $\{1, \ldots, n\}$. We deduce (47) by linearity.

Acknowledgement. The author thanks Geoffrey Grimmett for drawing his attention to the article [2], and the two anonymous referees for their helpful comments, corrections and suggestions.

\section{REFERENCES}

[1] M. Aizenman. Geometric analysis of $\phi^{4}$ fields and Ising models. Communications in Mathematical Physics, 86:1-48, 1982.

[2] M. Aizenman, H. Duminil-Copin, and V. Sidoravicius. Random Currents and Continuity of Ising Model's Spontaneous Magnetization. arXiv preprint arXiv:1311.1937, 2013.

[3] M. Aizenman and R. Fernández. On the critical behavior of the magnetization in high-dimensional Ising models. Journal of Statistical Physics, 44:393-454, 1986.

[4] M. Aizenman, A. Klein, and C. M. Newman. Percolation methods for disordered quantum Ising models. In R. Kotecký, editor, Phase Transitions: Mathematics, Physics, Biology. World Scientific, Singapore, 1992.

[5] M. Aizenman and B. Nachtergaele. Geometric aspects of quantum spin states. Communications in Mathematical Physics, 164:17-63, 1994.

[6] P. Billingsley. Convergence of probability measures. John Wiley \& Sons, 2009.

[7] J. E. Björnberg. Graphical representations of Ising and Potts models. PhD thesis, Cambridge and KTH, 2009. arXiv:1011.2683.

[8] J. E. Björnberg. Infrared bound and mean-field behaviour in the quantum Ising model. Communications in Mathematical Physics 323(1): 329-366, 2013.

[9] J. E. Björnberg and G. R. Grimmett. The phase transition of the quantum Ising model is sharp. Journal of Statistical Physics, 136(2):231, 2009.

[10] D. Burago, Y. Burago and S. Ivanov. A course in metric geometry. American Mathematical Society, 2001.

[11] R. M. Burton and M. Keane. Density and uniqueness in percolation. Communications in Mathematical Physics 121(3): 501-505, 1989.

[12] M. Campanino, A. Klein, and J. F. Perez. Localization in the ground state of the Ising model with a random transverse field. Communications in Mathematical Physics 135(3): 499-515, 1991.

[13] N. Crawford and D. Ioffe. Random current representation for transverse field Ising model. Communications in Mathematical Physics, 296:447-474, 2010.

[14] D. J. Daley and D. Vere-Jones. An introduction to the theory of point processes. Vols. 1 and 2. New York: Springer, 2005.

[15] W. Driessler, L. Landau, and J. F. Perez. Estimates of critical lengths and critical temperatures for classical and quantum lattice systems. Journal of Statistical Physics, 20(2): 123-162, 1979.

[16] F. J. Dyson, E. H. Lieb, and B. Simon. Phase transitions in quantum spin systems with isotropic and nonisotropic interactions. Journal of Statisical Physics, 18(4):335-383, 1978.

[17] C. M. Fortuin and P. W. Kasteleyn. On the random-cluster model: I. Introduction and relation to other models. Physica 57(4): 536-564, 1972. 
[18] J. Fröhlich, B. Simon, and T. Spencer. Infrared bounds, phase transitions and continuous symmetry breaking. Communications in Mathematical Physics, 50(1):79-95, 1976.

[19] C. Goldschmidt, D. Ueltschi, and P. Windridge. Quantum Heisenberg models and their probabilistic representations. Entropy and the Quantum II, Contemp. Math 552: 177-224, 2011.

[20] D. Ioffe. Stochastic geometry of classical and quantum Ising models. In Methods of Contemporary Mathematical Statistical Physics, volume 1970 of Lecture Notes in Mathematics. Springer, Berlin, 2009.

[21] J. L. Lebowitz and A. Martin-Löf. On the uniqueness of the equilibrium state for Ising spin systems. Communications in Mathematical Physics 25(4): 276$282,1972$.

[22] E. Lieb, T. Schultz, and D. Mattis. Two soluble models of an antiferromagnetic chain. Annals of Physics, 16:407-466, 1961.

[23] L. Onsager. Crystal statistics. I. A two-dimensional model with an orderdisorder transition. Physical Review 65(3-4):117, 1944.

[24] P. Pfeuty. The one-dimensional Ising model with a transverse field. Annals of Physics 57(1): 79-90, 1970 .

[25] B. Tóth. Improved lower bound on the thermodynamic pressure of the spin 1/2 Heisenberg ferromagnet. Letters in Mathematical Physics 28(1): 75-84, 1993.

[26] D. Ueltschi. Random loop representations for quantum spin systems. Journal of Mathematical Physics 54(8): 083301, 2013. 